\title{
Thermoelectric power in carbon nanotubes and quantum wires of nonlinear optical, optoelectronic, and related materials under strong magnetic field: Simplified theory and relative comparison
}

\author{
K. P. Ghatak ${ }^{\text {a) }}$ \\ Department of Electronic Science, The University of Calcutta, 92, Acharyya Prafulla Road, Kolkata 700 \\ 009, India \\ S. Bhattacharya \\ Department of Computer Science, St. Xavier's College, 30 Park Street, Kolkata 700 016, India
}

S. Bhowmik and R. Benedictus

Faculty of Aerospace Engineering, Delft University of Technology, Kluyverweg 1, 2629 HS Delft, The Netherlands

S. Choudhury

Department of Electronics and Communication Engineering, Sikkim Manipal Institute of Technology, Majitar, Rangpo, East Sikkim 737 132, India

(Received 10 October 2007; accepted 27 October 2007; published online 6 February 2008)

\begin{abstract}
We study thermoelectric power under strong magnetic field (TPM) in carbon nanotubes (CNTs) and quantum wires (QWs) of nonlinear optical, optoelectronic, and related materials. The corresponding results for QWs of III-V, ternary, and quaternary compounds form a special case of our generalized analysis. The TPM has also been investigated in QWs of II-VI, IV-VI, stressed materials, $n$-GaP, $p$ - $\mathrm{PtSb}_{2}, n$-GaSb, and bismuth on the basis of the appropriate carrier dispersion laws in the respective cases. It has been found, taking QWs of $n$-CdGeAs $2, n-\mathrm{Cd}_{3} \mathrm{As}_{2}, n$-InAs, $n$-InSb, $n$-GaAs, $n$ - $\mathrm{Hg}_{1-x} \mathrm{Cd}_{x} \mathrm{Te}, n-\mathrm{In}_{1-x} \mathrm{Ga}_{x} \mathrm{As}_{y} \mathrm{P}_{1-y}$ lattice-matched to InP, $p$-CdS, $n$-PbTe, $n$-PbSnTe, $n$ - $\mathrm{Pb}_{1-x} \mathrm{Sn}_{x} \mathrm{Se}$, stressed $n$-InSb, $n$-GaP, $p$-PtSb ${ }_{2}, n$-GaSb, and bismuth as examples, that the respective TPM in the QWs of the aforementioned materials exhibits increasing quantum steps with the decreasing electron statistics with different numerical values, and the nature of the variations are totally band-structure-dependent. In CNTs, the TPM exhibits periodic oscillations with decreasing amplitudes for increasing electron statistics, and its nature is radically different as compared with the corresponding TPM of QWs since they depend exclusively on the respective band structures emphasizing the different signatures of the two entirely different one-dimensional nanostructured systems in various cases. The well-known expression of the TPM for wide gap materials has been obtained as a special case under certain limiting conditions, and this compatibility is an indirect test for our generalized formalism. In addition, we have suggested the experimental methods of determining the Einstein relation for the diffusivity-mobility ratio and the carrier contribution to the elastic constants for materials having arbitrary dispersion laws. (C) 2008 American Institute of Physics. [DOI: 10.1063/1.2827365]
\end{abstract}

\section{INTRODUCTION}

Since Iijima's discovery, ${ }^{1}$ carbon nanotubes (CNTs) have been recognized as fascinating materials with nanometer dimensions uncovering new phenomena in different areas of low-dimensional science and technology. The remarkable physical properties of these quantum materials make them ideal candidates to reveal new phenomena in nanoelectronics. The CNTs find wide applications in conductive $^{2,3}$ and high strength composites, ${ }^{4}$ chemical sensors, ${ }^{5}$ field emission displays, ${ }^{6,7}$ hydrogen storage media, ${ }^{8,9}$ nanotweezeres, ${ }^{10}$ nanogears ${ }^{11}$ nanocantilever devices, ${ }^{12}$ nanomotors ${ }^{13}$ and nanoelectronic devices. ${ }^{14,15}$ Single-walled carbon nanotubes (SWNTs) appear to be excellent materials for single molecule electronics, ${ }^{16-18}$ nano-

\footnotetext{
${ }^{a)}$ Author to whom correspondence should be addressed. Electronic mail: kamakhyaghatak@yahoo.co.in.
}

tube based diodes, ${ }^{19}$ single electron transistors,${ }^{20}$ random access memory cells, ${ }^{21}$ logic circuits, ${ }^{22}$ and in other nanosystems. In this context, it may be noted that with the advent of molecular-beam epitaxy, fine line lithography, and other experimental techniques, low-dimensional structures having quantum confinement of one, two, and three dimensions (such as quantum wells, wires, and dots) have attracted much attention not only for their potential in uncovering new phenomena in nanoscience, but also for their interesting applications in nanotechnology. ${ }^{23-25}$

In QWs, the restriction of the motion of the carriers in the two directions may be viewed as carrier confinement by two infinitely deep one-dimensional (1D) rectangular potential wells, leading to quantization (known as quantizing size effect) of the wave vectors along the two orthogonal directions, allowing 1D electron transport representing new physical features not exhibited in bulk semiconductors. The lowdimensional heterostructures based on various materials are 
widely investigated because of the enhancement of carrier mobility. An enormous range of important applications in the quantum regime, together with a rapid increase in computing power, have generated much interest in the analysis of nanostructured devices for investigating their properties. ${ }^{26-29} \mathrm{Ex}$ amples of such new applications include various quantum wires, ${ }^{30-34}$ quantum resistors, ${ }^{35}$ resonant tunneling diodes and band filters, ${ }^{36,37}$ quantum switches, ${ }^{38}$ quantum sensors, ${ }^{39-41}$ quantum logic gates, ${ }^{42,43}$ quantum transistors and subtuners, ${ }^{44-46}$ heterojunction field-effect transistors (FETs),${ }^{47}$ high-speed digital networks, ${ }^{48}$ high-frequency microwave circuits, ${ }^{49}$ optical modulators, ${ }^{50}$ optical switching systems, ${ }^{51}$ and other devices. Though extensive work has already been done for both the CNTs and QWs, it appears from the literature that the TPM for both the CNTs and the QWs has yet to be investigated in detail.

It is well known that the TPM is a very important relation $^{52}$ since the entropy (a very important thermodynamic property that cannot be experimentally determined) can be known from this relation by knowing the experimental values of the electron concentration. The TPM is more accurate than any two of the individual relations for the electron concentration or the entropy, which is considered to be the two most widely used quantities in investigating the thermodynamics of the electronic materials. Besides, in recent years with the advent of the quantum Hall effect, ${ }^{53}$ there has been considerable interest in studying the TPM for various compounds having different band structures. ${ }^{54-63}$ It is worth remarking that the analysis of the thermopower generates information about the band structure, the density-of-states function, and the effective mass of the carriers. ${ }^{59}$ The classical TPM $(G)$ equation is given by $G=\left(\pi^{2} k_{B} / 3 e\right)\left(k_{B}\right.$ and $e$ are Boltzmann's constant and the magnitude of the carrier charge, respectively) and is well known in the literature. ${ }^{59}$ In this conventional form, it appears that the TPM depends only on the fundamental constants and is independent of temperature and carrier concentration under the condition of carrier nondegeneracy. Askerov et al. ${ }^{63}$ showed that the TPM is independent of scattering mechanisms and depends only on the dispersions laws of the carriers.

It is well known from the fundamental work of Zawadzki $^{61}$ that the TPM for electronic materials having degenerate electron concentration is essentially determined by their respective energy band structures. It has, therefore, different values in different materials and varies with the doping, the magnitude of the reciprocal quantizing magnetic field under magnetic quantization, the quantizing electric field as in inversion layers, the nanothickness as in quantum wells and quantum-well wires, and with the superlattice period as in quantum confined superlattices of small gap semiconductors with graded interfaces having various carrier energy spectra. Some of the significant features that have emerged from these studies are as follows:

(a) The TPM decreases monotonically with the increase in electron concentration.

(b) The TPM decreases with doping in heavily doped semiconductors forming band tails.

(c) The nature of variations is significantly influenced by the spectrum constants of various materials having different band structures.

(d) The TPM oscillates with inverse quantizing magnetic field due to the Shubnikov-de Haas effect.

(e) The TPM decreases with the magnitude of the quantizing electric field in inversion layers.

(f) The TPM exhibits composite oscillations with significantly different values in superlattices and various other quantized structures.

In this article, we have studied the TPM in CNTs and also in QWs of nonlinear optical, III-V, ternaries, quaternaries, II-VI, IV-VI, stressed materials, $n$-GaP, $p$ - $\mathrm{PtSb}_{2}$, $n$-GaSb, and bismuth on the basis of their respective carrier energy spectra. In this context, it may be noted that the nonlinear optical materials are also known as tetragonal compounds due to their crystal structure. ${ }^{64}$ These materials are being used increasingly in light-emitting diodes, Hall pickups, and thermal detectors. ${ }^{65-67}$ Rowe and Shay ${ }^{68}$ demonstrated that the quasicubic model ${ }^{69}$ can be used to explain the observed splitting and symmetry properties of the conduction and valence bands at the zone center of the $\mathbf{k}$ space of the aforementioned compounds. The $s$-like conduction band is singly degenerate and the $p$-like valence bands are triply degenerate. The latter splits into three subbands because of the spin-orbit and the crystal-field interactions. The large contribution of the crystal-field splitting occurs from the noncubic potential. $^{70}$ The experimental data on the absorption constants, ${ }^{71}$ the effective mass, ${ }^{72}$ and the optical third-order susceptibility ${ }^{73}$ have produced strong evidence that the conduction band in the same compound corresponds to a single ellipsoid of revolution at the zone center in $\mathbf{k}$ space.

Considering the crystal potential in the Hamiltonian, and special features of the nonlinear optical compounds, Kildal ${ }^{74}$ proposed the energy spectrum of the conduction electrons under the assumptions of the isotropic momentum matrix element and the isotropic spin orbit splitting constant, respectively, although the anisotropies in the two aforementioned band parameters are the significant physical features of said compounds. ${ }^{75}$

In Sec. II A, we have formulated the expressions of the TPM in CNTs by formulating the respective expressions of the electron statistics for both $(n, n)$ and $(n, 0)$ tubes, respectively. In Sec. II B, we have studied the TPM in QWs of nonlinear optical materials by formulating the generalized dispersion relation of the conduction electrons, considering the anisotropies of the effective electron masses and the spinorbit splitting of the valance band together with the proper inclusion of crystal-field splitting in the Hamiltonian through the $k \cdot p$ formalism. In Sec. II C, it has been shown that the corresponding results for the QWs of III-V, ternary, and quaternary materials form special cases of our generalized analysis as derived in Sec. II B. The III-V materials are being used increasingly in integrated optoelectronics, ${ }^{76}$ passive filter devices, ${ }^{77}$ distributed feedback lasers, and Bragg reflectors. ${ }^{78}$ We have used $n-\mathrm{Hg}_{1-x} \mathrm{Cd}_{x} \mathrm{Te}$ and $n-\mathrm{In}_{1-x} \mathrm{Ga}_{x} \mathrm{As}_{y} \mathrm{P}_{1-y}$ lattice matched to InP as examples of ternary and quaternary compounds, respectively. The $n-\mathrm{Hg}_{1-x} \mathrm{Cd}_{x} \mathrm{Te}$ is a classic narrowgap compound and is an important optoelectronic material 
because its band gap can be varied to cover a spectral range from $0.8 \mu \mathrm{m}$ to over $30 \mu \mathrm{m}$ by adjusting the alloy composition. ${ }^{79}$ This compound finds extensive applications in infrared detector materials ${ }^{80}$ and photovoltaic detector arrays ${ }^{81}$ in the $8-12 \mu \mathrm{m}$ wave bands. The above uses have spurred an $\mathrm{Hg}_{1-x} \mathrm{Cd}_{x} \mathrm{Te}$ technology for the production of high-mobility single crystals, with specially prepared surface layers that are also ideally suitable for narrow subband physics because the relevant material constants are within the reach of experiment. ${ }^{82}$ The quaternary compounds also find extensive applications in optoelectronics, high electron mobility transistors, visible heterostructure compound lasers, infrared light-emitting diodes, lasers for fiber optic systems, ${ }^{83}$ tandem solar cells, ${ }^{84}$ avalanche photodetectors, ${ }^{85}$ longwavelength light sources, detectors in optical fiber communications, ${ }^{86}$ and new types of optical devices. ${ }^{87}$ The expressions for electron concentration per unit length $\left(n_{1 \mathrm{D}}\right)$ and the TPM for QWs whose energy band structures are defined by the two-band model of Kane and that of parabolic energy bands have further been formulated under certain limiting conditions for the purpose of relative assessment. To perform numerical computations, $n-\mathrm{CdGeAs}_{2}$ and $n-\mathrm{Cd}_{3} \mathrm{As}_{2}$ have been used as examples of nonlinear optical and tetragonal compounds. ${ }^{88}$ The TPM has also been investigated numerically by taking $n$-InAs, $n$-InSb, and $n$-GaAs as examples of III-V compounds and $n-\mathrm{Hg}_{1-x} \mathrm{Cd}_{x} \mathrm{Te}$ and $n-\mathrm{In}_{1-x} \mathrm{Ga}_{x} \mathrm{As}_{y} \mathrm{P}_{1-y}$ lattice-matched to InP as examples of ternary and quaternary materials in accordance with the threeand the two-band models of Kane together with the parabolic energy bands, respectively, for the purpose of relative comparison among the above-mentioned models.

The II-VI compounds find extensive applications in infrared detectors, ${ }^{89}$ ultrahigh-speed bipolar transistors, ${ }^{90}$ optic fiber communications, ${ }^{91}$ and advanced microwave devices. ${ }^{92}$ These compounds possess the appropriate direct band gap to produce light-emitting diodes and lasers from blue to red wavelengths. The Hopfield model describes the dispersion relation of both the carriers of II-VI materials where the splitting of the two-spin states by the spin-orbit coupling and the crystalline field has been taken into account. ${ }^{93}$ In Sec. II D, we shall study the TPM in QWs of II-VI compounds on the basis of the Hopfield model by formulating the appropriate carrier statistics and taking $\mathrm{CdS}$ as an example.

In Sec. II E, we shall study the TPM in QWs of IV-VI materials, which are being widely used in thermoelectric devices, superlattices, and other quantum effect devices. ${ }^{94}$ The dispersion relation of the carriers in IV-VI compounds has been formulated by Dimmock ${ }^{94}$ by including the contributions of the transverse and longitudinal effective masses of the external bands, which arises from the $\mathbf{k} \cdot \mathbf{p}$ perturbations with the other bands, taken to the second order together with the special anisotropic properties of the energy band structures of the above-mentioned compounds. For the purpose of numerical computations, we have used $\mathrm{PbTe}, \mathrm{PbSnTe}$, and $\mathrm{Pb}_{1-x} \mathrm{Sn}_{x} \mathrm{Se}$ as examples of IV-VI compounds. In recent years, there has been considerable interest in studying the various electronic properties of stressed compounds because of their important physical features. ${ }^{95}$ In Sec. II F, we have formulated the TPM in QWs of stressed compounds, taking stressed $n$-InSb as an example.

The $n$-GaP, platinum antimonide, and gallium antimonide occupy significant positions in the realm of quantum effect devices due to their important physical properties and the corresponding dispersion relations. ${ }^{96,110,111}$ We have studied the TPM for the QWs of $n$-GaP, $p$ - $\mathrm{PtSb}_{2}$, and $n$-GaSb in Secs. II G, II H, and II I, respectively, by considering the appropriate carrier energy spectra.

It is well known that the carrier energy spectra in bismuth differ considerably from simple spherical surfaces of the degenerate electron gas, and several models have been developed to describe the energy band structure of Bi. Earlier works ${ }^{97,98}$ demonstrated that the ellipsoidal parabolic model or the one-band model could describe the carrier properties of Bi. Shoenberg ${ }^{97}$ indicated that the de Haas-Van Alphen and cyclotron resonance experiments supported the one-band model, although the latter work showed that $\mathrm{Bi}$ could be described by the two-band nonparabolic ellipsoidal Lax model since the magnetic field dependence of many physical parameters of Bi supports the above model. ${ }^{99}$ The magnetooptical results ${ }^{100}$ and the ultrasonic quantum oscillation data ${ }^{101}$ favor the Lax ellipsoidal nonparabolic model, ${ }^{102}$ whereas Kao, ${ }^{102}$ Dinger and Lawson, ${ }^{103}$ and Koch and Jensen ${ }^{104}$ indicated that the Cohen model ${ }^{105}$ is in better agreement with the experimental results. It may be noted that the Hybrid model of Bi as proposed by Takaoka et al. ${ }^{106}$ also explains many important physical properties. Besides, McClure and $\mathrm{Choi}^{107}$ presented a new model of Bi that fits the data for a large number of magneto-oscillatory and resonance experiments. In Sec. II J, we have formulated the TPM in QWs of $\mathrm{Bi}$ in accordance with the aforementioned energy band models for the purpose of relative assessment. In the Sec. II K, we have suggested the experimental methods of determination of the Einstein relation for the diffusivitymobility ratio and the carrier contribution to the elastic constants for materials having arbitrary carrier energy spectra.

\section{THEORETICAL BACKGROUND}

\section{A. Investigation of the TPM in $(n, n)$ and $(n, 0)$ CNTs}

For the $(n, n)$ and $(n, 0)$ tubes, the energy dispersion relations are given by ${ }^{108}$

$$
\begin{aligned}
& E_{m}\left(k_{y}\right)= \pm t \sqrt{1+4 \cos \frac{m \pi}{n} \cos \frac{k_{y} a}{2}+4 \cos ^{2} \frac{k_{y} a}{2}}, \\
& -\frac{\pi}{a}<k_{y}<\frac{\pi}{a}
\end{aligned}
$$

and

$$
E_{m}\left(k_{x}\right)= \pm t \sqrt{1+4 \cos \frac{\sqrt{3} k_{x} a}{n} \cos \frac{m \pi}{n}+4 \cos ^{2} \frac{m \pi}{n}}
$$




$$
-\frac{\pi}{\sqrt{3} a}<k_{x}<\frac{\pi}{\sqrt{3} a}
$$

where the notations are defined in Refs. 108 and 109. Using Eqs. (1) and (2), the electron concentration per unit length $\left(n_{1 \mathrm{D}}\right)$ can be written as

$$
n_{1 \mathrm{D}}=\frac{4}{\pi a}\left[A_{1}\left(E_{F}, m, n\right)+B_{1}\left(E_{F}, m, n\right)\right]
$$

and

$$
n_{1 \mathrm{D}}=\frac{4}{a \pi \sqrt{3}}\left[A_{2}\left(E_{F}, m, n\right)+B_{2}\left(E_{F}, m, n\right)\right],
$$

where

$$
\begin{aligned}
& A_{1}\left(E_{F}, m, n\right)=\cos ^{-1}\left[\frac{1}{2}\left(\sqrt{A^{2}-1+\left(E_{F} / t\right)^{2}}-A\right)\right], \\
& A=\cos \left(\frac{m \pi}{n}\right)
\end{aligned}
$$

$E_{F}$ is the Fermi energy,

$$
B_{1}\left(E_{F}, m, n\right)=\sum_{r=1}^{S_{0}} Z_{r} A_{1}\left(E_{F}, m, n\right),
$$

$r$ is the set of real positive integers whose upper limit is $S_{0}$,

$$
Z_{r}=2\left(k_{B} T\right)^{2 r}\left(1-2^{1-2 r}\right) \xi(2 r) \frac{\partial^{2 r}}{\partial E_{F}^{2 r}},
$$

$T$ is the temperature, $\xi(2 r)$ is the zeta function of order $2 r$,

$$
A_{2}\left(E_{F}, m, n\right)=\cos ^{-1}\left[(4 A)^{-1}\left\{\left(\frac{E_{F}}{t}\right)^{2}-1-4 A^{2}\right\}\right],
$$

and

$$
B_{2}\left(E_{F}, m, n\right)=\sum_{r=1}^{S_{0}} Z_{r} A_{2}\left(E_{F}, m, n\right) .
$$

The TPM in this case can, in general, be expressed as ${ }^{60}$

$$
G=\frac{\pi^{2} k_{B}^{2} T}{3 e n_{1 \mathrm{D}}}\left(\frac{\partial n_{1 \mathrm{D}}}{\partial E_{F}}\right) \text {. }
$$

Combining Eqs. (3)-(5), the expressions of the TPM for the $(n, n)$ and $(n, 0)$ tubes can be written as

$$
G=\frac{\pi^{2} k_{B}^{2} T}{3 e}\left(\frac{A_{1}^{\prime}\left(E_{F}, m, n\right)+B_{1}^{\prime}\left(E_{F}, m, n\right)}{A_{1}\left(E_{F}, m, n\right)+B_{1}\left(E_{F}, m, n\right)}\right)
$$

and

$$
G=\frac{\pi^{2} k_{B}^{2} T}{3 e}\left(\frac{A_{2}^{\prime}\left(E_{F}, m, n\right)+B_{2}^{\prime}\left(E_{F}, m, n\right)}{A_{2}\left(E_{F}, m, n\right)+B_{2}\left(E_{F}, m, n\right)}\right),
$$

where the primes denote the differentiation of the respective differentiable functions with respect to $E_{F}$.

\section{B. Investigation of the TPM in QWs of nonlinear optical materials}

The form of the $\boldsymbol{k} \cdot \boldsymbol{p}$ matrix for the nonlinear optical materials can be written as

$$
H=\left[\begin{array}{ll}
H_{1} & H_{2} \\
H_{2}^{+} & H_{1}
\end{array}\right],
$$

where

$$
H_{1}=\left[\begin{array}{cccc}
E_{g} & 0 & P_{\|} k_{z} & 0 \\
0 & \left(-2 \Delta_{\perp} / 3\right) & \left(\sqrt{2} \Delta_{\perp} / 3\right) & 0 \\
P_{\|} k_{z} & \left(\sqrt{2} \Delta_{\perp} / 3\right) & -\left(\delta+\frac{1}{3} \Delta_{\|}\right) & 0 \\
0 & 0 & 0 & 0
\end{array}\right]
$$

and

$$
H_{2}=\left[\begin{array}{cccc}
0 & -f_{,+} & 0 & f_{,-} \\
f_{,+} & 0 & 0 & 0 \\
0 & 0 & 0 & 0 \\
f_{,+} & 0 & 0 & 0
\end{array}\right],
$$

in which $E_{g}$ is the band gap, $P_{\|}$and $P_{\perp}$ are the momentum matrix elements parallel and perpendicular to the direction of the $c$-axis, respectively, $\delta$ is the crystal-field splitting constant, $\Delta_{\|}$and $\Delta_{\perp}$ are the spin-orbit splitting constants parallel and perpendicular to the direction of the $c$ axis, respectively, $f_{ \pm}=\left(P_{\perp} / \sqrt{2}\right)\left(k_{x} \pm i k_{y}\right)$, and $i=\sqrt{-1}$.

The diagonalization of the above matrix leads to the expression of the electron dispersion law in bulk specimens of nonlinear materials as

$$
\gamma(E)=f_{1}(E) k_{s}^{2}+f_{2}(E) k_{z}^{2},
$$

where

$$
\begin{aligned}
\gamma(E)= & \left\{E ( E + E _ { g } ) \left[\left(E+E_{g}\right)\left(E+E_{g}+\Delta_{\|}\right)+\delta\left(E+E_{g}\right.\right.\right. \\
& \left.\left.\left.+\frac{1}{3} \Delta_{\|}\right)\right]+\frac{2}{9} E\left(E+E_{g}\right)\left(\Delta_{\|}^{2}-\Delta_{\perp}^{2}\right)\right\}, \\
f_{1}(E)= & \left\{\hbar^{2} E_{g}\left(E_{g}+\Delta_{\perp}\right)\left[2 m_{\perp}^{*}\left(E_{g}+\frac{2}{3} \Delta_{\perp}\right)\right]^{-1}\right\}\left[\delta \left(E+E_{g}\right.\right. \\
& \left.+\frac{1}{3} \Delta_{\|}\right)+\left(E+E_{g}\right)\left(E+E_{g}+\frac{2}{3} \Delta_{\|}\right)+\frac{1}{9}\left(\Delta_{\|}^{2}\right. \\
& \left.\left.-\Delta_{\perp}^{2}\right)\right], \quad k_{s}^{2}=k_{x}^{2}+k_{y}^{2}, \\
f_{2}(E)= & \left\{\hbar^{2} E_{g}\left(E_{g}+\Delta_{\|}\right)\left[2 m_{\|}^{*}\left(E_{g}+\frac{2}{3} \Delta_{\|}\right)\right]^{-1}\right\} \\
& \times\left[\left(E+E_{g}\right)\left(E+E_{g}+\frac{2}{3} \Delta_{\|}\right)\right],
\end{aligned}
$$

$E$ is the electron energy measured from the edge of the conduction band in the vertically upward direction in the absence of any quantization, $\hbar \equiv h / 2 \pi, h$ is Planck's constant, and

$m_{\|}^{*}$ and $m_{\perp}^{*}$ are the effective electron masses at the edge of the conduction band parallel and perpendicular to the direction of the $c$ axis, respectively.

The 1D electron energy spectrum in QWs of nonlinear optical compounds can be expressed as

$$
\gamma(E)=f_{1}(E) \phi\left(n_{x}, n_{y}\right)+f_{2}(E) k_{z}^{2},
$$

where $\phi\left(n_{x}, n_{y}\right)=\left(n_{x} \pi / d_{x}\right)^{2}+\left(n_{y} \pi / d_{y}\right)^{2}, n_{x}(=1,2,3, \ldots)$, and $n_{y}(=1,2,3, \ldots)$ are the size quantum numbers along the $x$ and $y$ directions, respectively, and $d_{x}$ and $d_{y}$ are the nanothickness along the respective directions.

The carrier statistics in this case can be expressed as 


$$
n_{1 D}=\frac{2}{\pi} \sum_{n_{x}=1}^{n_{x_{\max }}} \sum_{n_{y}=1}^{n_{y_{\max }}}\left[T_{1}\left(E_{F}, n_{x}, n_{y}\right)+T_{2}\left(E_{F}, n_{x}, n_{y}\right)\right],
$$

where

$$
\begin{aligned}
& T_{1}\left(E_{F}, n_{x}, n_{y}\right) \equiv\left(\frac{\gamma_{1}\left(E_{F}\right)-f_{1}\left(E_{F}\right) \phi\left(n_{x}, n_{y}\right)}{f_{2}\left(E_{F}\right)}\right)^{1 / 2}, \\
& T_{2}\left(E_{F}, n_{x}, n_{y}\right) \equiv \sum_{r=1}^{s_{o}} Z_{r}\left[T_{1}\left(E_{F}, n_{x}, n_{y}\right)\right] .
\end{aligned}
$$

Therefore, combining Eqs. (5) and (13), we get

$$
G=\left(\frac{\pi^{2} k_{B}^{2} T}{3 e} \sum_{n_{x}=1}^{\sum_{n_{x}=1}^{n_{x_{\max }}} \sum_{n_{y}=1}^{n_{n_{\max }}} \sum_{n_{x_{\max }}=1}^{n_{y_{\max }}}\left[T_{1}^{\prime}\left(E_{F}, n_{x}, n_{y}\right)+T_{2}^{\prime}\left(E_{F}, n_{x}, n_{y}, n_{y}\right)+T_{2}\left(E_{F}, n_{x}, n_{y}\right)\right]} .\right.
$$

\section{Investigation of TPM for QWs of III-V, ternary, and quaternary materials}

(a) Under the conditions $\Delta_{\|}=\Delta_{\perp}=\Delta$ (the isotropic spin orbiting constant), $\delta=0$, and $m_{\|}^{*}=m_{\perp}^{*}=m^{*}$ (the isotropic effective electron mass at the edge of the conduction band), Eq. (11) assumes the form

$$
\frac{\hbar^{2} k^{2}}{2 m^{*}}=I(E), \quad I(E) \equiv \frac{E\left(E+E_{g}\right)\left(E+E_{g}+\Delta\right)\left(E_{g}+\frac{2}{3} \Delta\right)}{E_{g}\left(E_{g}+\Delta\right)\left(E+E_{g}+\frac{2}{3} \Delta\right)} .
$$

Equation (15) describes the dispersion relation of the conduction electrons in III-V, ternary, and quaternary materials and is well known in the literature as the three-band model of Kane, which should be used as such for studying the electronic properties of such compounds where the spinorbit splitting constant is of the order of the band gap. ${ }^{109}$

The 1D dispersion relation in this case is given by

$$
\frac{\hbar^{2} \phi\left(n_{x}, n_{y}\right)}{2 m^{*}}+\frac{\hbar^{2} k_{z}^{2}}{2 m^{*}}=I(E)
$$

The 1D electron statistics can thus be written as

$$
n_{1 D}=\left(\frac{2 \sqrt{2 m^{*}}}{\pi \hbar}\right) \sum_{n_{x}=1}^{n_{x_{\max }}} \sum_{n_{y}=1}^{n_{y_{\max }}}\left[T_{3}\left(E_{F}, n_{x}, n_{y}\right)+T_{4}\left(E_{F}, n_{x}, n_{y}\right)\right] \text {, }
$$

where

$$
T_{3}\left(E_{F}, n_{x}, n_{y}\right) \equiv\left(I\left(E_{F}\right)-\frac{\hbar^{2}}{2 m^{*}} \phi\left(n_{x}, n_{y}\right)\right)^{1 / 2}
$$

and

$$
T_{4}\left(E_{F}, n_{x}, n_{y}\right) \equiv \sum_{r=1}^{s_{0}} Z_{r} T_{3}\left(E_{F}, n_{x}, n_{y}\right) .
$$

The use of Eqs. (5) and (17) leads to the expression of the TPM in this case as

$$
G=\left(\frac{\pi^{2} k_{B}^{2} T}{3 e}\right) \frac{\sum_{n_{x}=1}^{n_{x_{\max }}} \sum_{n_{y}=1}^{n_{y_{\max }}}\left[T_{3}^{\prime}\left(E_{F}, n_{x}, n_{y}\right)+T_{4}^{\prime}\left(E_{F}, n_{x}, n_{y}\right)\right]}{\sum_{n_{x}=1} \sum_{n_{\max }}^{n_{y_{\max }}}\left[T_{3}\left(E_{F}, n_{x}, n_{y}\right)+T_{4}\left(E_{F}, n_{x}, n_{y}\right)\right]} .
$$

(b) Under the inequalities $\Delta \gg E_{g}$ or $\Delta \ll E_{g}$, Eq. (15) assumes the form

$$
E\left(1+\beta_{0} E\right)=\frac{\hbar^{2} k^{2}}{2 m^{*}}, \quad \beta_{0} \equiv 1 / E_{g} .
$$

Equation (19) is known as the two-band model of Kane and should be as such for studying the electronic properties of such Kane materials (e.g., InSb) whose energy band structures satisfy the aforementioned constraints.

The 1D electron statistics in this case is given by

$$
n_{1 \mathrm{D}}=\left(\frac{2 \sqrt{2 m^{*}}}{\pi \hbar}\right)^{n_{x}=1} \sum_{n_{y}=1}^{n_{x_{\max }}} \sum_{n_{\max }}^{n_{y}}\left[T_{5}\left(E_{F}, n_{x}, n_{y}\right)+T_{6}\left(E_{F}, n_{x}, n_{y}\right)\right],
$$

where

$$
T_{5}\left(E_{F}, n_{x}, n_{y}\right) \equiv\left(E_{F}\left(1+\beta_{0} E_{F}\right)-\frac{\hbar^{2}}{2 m^{*}} \phi\left(n_{x}, n_{y}\right)\right)^{1 / 2}
$$

and

$$
T_{6}\left(E_{F}, n_{x}, n_{y}\right) \equiv \sum_{r=1}^{s_{0}} Z_{r} T_{5}\left(E_{F}, n_{x}, n_{y}\right) .
$$

The use of Eqs. (5) and (20) leads to the expression of the TPM in this case as

$$
G=\left(\frac{\pi^{2} k_{B}^{2} T}{3 e}\right) \frac{\sum_{n_{x}=1}^{n_{x_{\max }}} \sum_{n_{y}=1}^{n_{y_{\max }}}\left[T_{5}^{\prime}\left(E_{F}, n_{x}, n_{y}\right)+T_{6}^{\prime}\left(E_{F}, n_{x}, n_{y}\right)\right]}{\sum_{n_{x}=1} \sum_{n_{\max }=1}^{n_{y_{\max }}}\left[T_{5}\left(E_{F}, n_{x}, n_{y}\right)+T_{6}\left(E_{F}, n_{x}, n_{y}\right)\right]} .
$$

(c) For $\beta_{0} \rightarrow 0$, Eq. (19) assumes the well-known form of the electron dispersion law of wide-gap materials as

$$
E=\frac{\hbar^{2} k^{2}}{2 m^{*}} .
$$

Thus under the condition $\beta_{0} \rightarrow 0$, the expressions of $n_{1 \mathrm{D}}$ and TPM for QWs in this case can be written from Eqs. (20) and (21) respectively, as

$$
n_{1 \mathrm{D}}=2\left(\frac{\sqrt{2 \pi m^{*} k_{B} T}}{h}\right) \sum_{n_{x}=1}^{n_{x_{\max }}} \sum_{n_{y}=1}^{n_{y_{\max }}}\left[F_{-1 / 2}\left(\eta_{1}\right)\right]
$$




$$
G=\left(\frac{\pi^{2} k_{B}}{3 e}\right) \frac{\sum_{n_{x}=1}^{n_{x_{\max }}} \sum_{n_{y}=1}^{n_{y_{\max }}}\left[F_{-3 / 2}\left(\eta_{1}\right)\right]}{\sum_{n_{\max }} \sum_{n_{y}=1}^{n_{y_{\max }}}\left[F_{-1 / 2}\left(\eta_{1}\right)\right]},
$$

where $\eta_{1}=\left(k_{B} T\right)^{-1}\left[E_{F}-\left\{\left(\hbar^{2} / 2 m^{*}\right) \phi\left(n_{x}, n_{y}\right)\right\}\right]$ and $F_{t}\left(\eta_{1}\right)$ is the one-parameter Fermi-Dirac integral of order $t_{0}$, which can be written as ${ }^{117}$

$$
\begin{aligned}
& F_{t_{0}}\left(\eta_{1}\right)=\left(\frac{1}{\Gamma\left(t_{0}+1\right)}\right) \int_{0}^{\infty} y^{t_{0}}\left[1+\exp \left(y-\eta_{1}\right)\right]^{-1} d y, \\
& y>-1,
\end{aligned}
$$

where $\Gamma\left(t_{0}+1\right)$ is the complete Gamma function for all $t_{0}$, analytically continued as a complex contour integral around the negative axis,

$$
F_{t_{0}}\left(\eta_{1}\right)=A_{t_{0}} \int_{(-\infty)}^{(0+)} y^{t_{0}}\left[1+\exp \left(-y-\eta_{1}\right)\right]^{-1} d y
$$

in which

$$
A_{t_{0}}=\frac{\Gamma\left(-t_{0}\right)}{2 \pi \sqrt{-1}} .
$$

Converting the summations over $n_{x}$ and $n_{y}$ to the corresponding integrations over the above-mentioned variables, from Eq. (24) we can write

$$
G=\frac{\pi^{2} k_{B}}{3 e} \frac{\left[F_{-1 / 2}\left(E_{F} / k_{B} T\right)\right]}{\left[F_{1 / 2}\left(E_{F} / k_{B} T\right)\right]} .
$$

Under the condition of carrier nondegeneracy, Eq. (27) gets simplified into the classical TPM equation as given in the Introduction.

\section{Investigation of TPM for QWs of II-VI materials}

The dispersion relation of the conduction electrons in bulk specimens of II-VI compounds in accordance with the Hopfield model can be expressed as ${ }^{93}$

$$
E=A_{o} k_{s}^{2}+B_{o} k_{z}^{2} \pm C_{o} k_{s},
$$

where $A_{o} \equiv \hbar^{2} / 2 m_{\perp}^{*}, B_{o} \equiv \hbar^{2} / 2 m_{\|}^{*}$, and $C_{o}$ represents the splitting of the two-spin states by the spin-orbit coupling and the crystalline field. For QWs of II-VI materials, the 1D dispersion relation assumes the form

$$
E=A_{o} \phi\left(n_{x}, n_{y}\right)+B_{o} k_{z}^{2} \pm C_{o} \sqrt{\phi\left(n_{x}, n_{y}\right)} .
$$

The use of Eq. (29) leads to the expression of the electron concentration per unit length as

$$
n_{1 \mathrm{D}}=\left(\frac{k_{B} T}{\pi B_{0}}\right)^{1 / 2} \sum_{n_{x}=1}^{n_{x_{\max }}} \sum_{n_{y}=1}^{n_{y_{\max }}}\left[F_{-1 / 2}\left(\eta_{2,+}\right)+F_{-1 / 2}\left(\eta_{2,-}\right)\right],
$$

where

$$
\eta_{2, \pm}=\left(k_{B} T\right)^{-1}\left\{E_{F}-\left[A_{o} \phi\left(n_{x}, n_{y}\right) \pm C_{o} \sqrt{\phi\left(n_{x}, n_{y}\right)}\right]\right\} .
$$

Using Eqs. (5) and (30), the TPM in QWs in this case can be expressed as

$$
G=\left(\frac{\pi^{2} k_{B}}{3 e}\right) \sum_{n_{x}=1}^{\sum_{n_{x}=1}^{n_{x_{\max }}} \sum_{n_{y}=1}^{n_{y_{\max }}}\left[F_{-3 / 2}\left(\eta_{2,+}\right)+F_{-3 / 2}\left(\eta_{2,-}\right)\right]} .
$$

Under the conditions $C_{o} \rightarrow 0$ and $m_{\|}^{*}=m_{\perp}^{*}=m^{*}$, Eq. (31) gets simplified to the well-known form as given by Eq. (24).

\section{E. Investigation of TPM for QWs of IV-VI compounds}

The dispersion relation of the conduction electrons in IV-VI materials can be expressed following Dimmock as ${ }^{94}$

$$
\begin{aligned}
( & \left.\in-\frac{E_{g}}{2}-\frac{\hbar^{2} k_{s}^{2}}{2 m_{t}^{-}}-\frac{\hbar^{2} k_{z}^{2}}{2 m_{l}^{-}}\right)\left(\in+\frac{E_{g}}{2}+\frac{\hbar^{2} k_{s}^{2}}{2 m_{t}^{+}}+\frac{\hbar^{2} k_{z}^{2}}{2 m_{l}^{+}}\right) \\
& =P_{\perp}^{2} k_{s}^{2}+P_{\|}^{2} k_{z}^{2},
\end{aligned}
$$

where $\in$ is the electron energy as measured from the center of the band gap $E_{g}$, and $m_{t}^{ \pm}$and $m_{l}^{ \pm}$represent the contributions of the transverse and longitudinal effective masses of the external $L_{6}^{+}$and $L_{6}^{-}$bands arising from the $\mathbf{k} \cdot \mathbf{p}$ perturbations with the other bands taken to the second order, respectively.

Substituting

$$
P_{\perp}^{2}=\frac{\hbar^{2} E_{g}}{2 m_{t}^{*}},
$$

$$
P_{\|}^{2}=\frac{\hbar^{2} E_{g}}{2 m_{l}^{*}},
$$

and

$$
\in=E+\frac{E_{g}}{2}
$$

(where $m_{t}^{*}$ and $m_{l}^{*}$ are the transverse and longitudinal effective masses at $k=0)$ in Eq. (32), we can write

$$
\begin{aligned}
(E & \left.-\frac{\hbar^{2} k_{s}^{2}}{2 m_{t}^{-}}-\frac{\hbar^{2} k_{z}^{2}}{2 m_{l}^{-}}\right)\left(1+\beta_{o} E+\beta_{o} \frac{\hbar^{2} k_{s}^{2}}{2 m_{t}^{+}}+\beta_{o} \frac{\hbar^{2} k_{z}^{2}}{2 m_{l}^{+}}\right) \\
& =\frac{\hbar^{2} k_{s}^{2}}{2 m_{t}^{*}}+\frac{\hbar^{2} k_{z}^{2}}{2 m_{l}^{*}} .
\end{aligned}
$$

The 1D dispersion relation in QWs of IV-VI compounds assumes the form 


$$
\begin{aligned}
E(1 & \left.+\beta_{0} E\right)+\beta_{0} E\left[\frac{x}{x_{4}}+\frac{\hbar^{2}}{2 x_{5}}\left(\frac{n_{y} \pi}{d_{y}}\right)^{2}\right]+\beta_{0} E \frac{\hbar^{2}}{2 x_{6}}\left(\frac{n_{z} \pi}{d_{z}}\right)^{2}-\left(1+\beta_{0} E\right)\left[\frac{x}{x_{1}}+\frac{\hbar^{2}}{2 x_{2}}\left(\frac{n_{y} \pi}{d_{y}}\right)^{2}\right]-\beta_{0}\left[\frac{x}{x_{1}}+\frac{\hbar^{2}}{2 x_{2}}\left(\frac{n_{y} \pi}{d_{y}}\right)^{2}\right]\left[\frac{x}{x_{4}}\right. \\
& \left.+\frac{\hbar^{2}}{2 x_{5}}\left(\frac{n_{y} \pi}{d_{y}}\right)^{2}\right]-\beta_{0}\left[\frac{x}{x_{1}}+\frac{\hbar^{2}}{2 x_{2}}\left(\frac{n_{y} \pi}{d_{y}}\right)^{2}\right] \frac{\hbar^{2}}{2 x_{6}}\left(\frac{n_{z} \pi}{d_{z}}\right)^{2}-\left(1+\beta_{0} E\right) \frac{\hbar^{2}}{2 x_{3}}\left(\frac{n_{z} \pi}{d_{z}}\right)^{2}-\beta_{0} \frac{\hbar^{2}}{2 x_{3}}\left(\frac{n_{z} \pi}{d_{z}}\right)^{2}\left[\frac{x}{x_{4}}+\frac{\hbar^{2}}{2 x_{5}}\left(\frac{n_{y} \pi}{d_{y}}\right)^{2}\right] \\
& -\beta_{0} \frac{\hbar^{2}}{2 x_{3}}\left(\frac{n_{z} \pi}{d_{z}}\right)^{2} \frac{\hbar^{2}}{2 x_{6}}\left(\frac{n_{z} \pi}{d_{z}}\right)^{2}=\frac{x}{m_{1}}+\frac{\hbar^{2}}{2 m_{2}}\left(\frac{n_{y} \pi}{d_{y}}\right)^{2}+\frac{\hbar^{2}}{2 m_{3}}\left(\frac{n_{z} \pi}{d_{z}}\right)^{2},
\end{aligned}
$$

where

$$
\begin{aligned}
& x=\frac{\hbar^{2} k_{x}^{2}}{2}, \\
& x_{4}=m_{t}^{+}, \\
& x_{5}=\frac{m_{t}^{+}+2 m_{l}^{+}}{3}, \\
& x_{6}=\frac{3 m_{t}^{+} m_{l}^{+}}{2 m_{l}^{+}+m_{t}^{+}} \\
& x_{1}=m_{t}^{-}, \\
& x_{2}=\frac{m_{t}^{-}+2 m_{l}^{-}}{3}, \\
& x_{3}=\frac{3 m_{t}^{-} m_{l}^{-}}{2 m_{l}^{-}+m_{t}^{-}},
\end{aligned}
$$

$$
\begin{aligned}
& m_{1}=m_{t}^{*}, \\
& m_{2}=\frac{m_{t}^{*}+2 m_{l}^{*}}{3},
\end{aligned}
$$

and

$$
m_{3}=\frac{3 m_{l}^{*} m_{t}^{*}}{m_{t}^{*}+2 m_{l}^{*}} .
$$

The use of Eq. (33) leads to the expression of $n_{1 \mathrm{D}}$ as

$$
n_{1 \mathrm{D}}=\frac{2 g_{v}}{\pi \hbar \sqrt{a_{1}}} \sum_{n_{y}=1}^{n_{y_{\max }}} \sum_{n_{z=1}}^{n_{z_{\max }}}\left[T_{7}\left(E_{F}, n_{y}, n_{z}\right)+T_{8}\left(E_{F}, n_{y}, n_{z}\right)\right] \text {, }
$$

where $g_{v}$ is the valley degeneracy,

$$
\begin{aligned}
T_{7}\left(E_{F}, n_{y}, n_{z}\right)= & {\left[\sqrt{b_{1}^{2}\left(E_{F}, n_{y}, n_{z}\right)+4 a_{1} c_{1}\left(E_{F}, n_{y}, n_{z}\right)}\right.} \\
& \left.-b_{1}\left(E_{F}, n_{y}, n_{z}\right)\right]^{1 / 2}, \\
a_{1}=\frac{\beta_{0}}{x_{1} x_{4}}, &
\end{aligned}
$$

$$
\begin{aligned}
b_{1}\left(E_{F}, n_{y}, n_{z}\right)= & \left\{\frac{-\beta_{0} E_{F}}{x_{4}}+\frac{1+\beta_{0} E_{F}}{x_{1}}+\beta_{0}\left[\frac{\hbar^{2}}{2 x_{2} x_{4}}\left(\frac{n_{y} \pi}{d_{y}}\right)^{2}+\frac{\hbar^{2}}{2 x_{1} x_{5}}\left(\frac{n_{y} \pi}{d_{y}}\right)^{2}\right]+\frac{\beta_{0} \hbar^{2}}{2 x_{1} x_{6}}\left(\frac{n_{z} \pi}{d_{z}}\right)^{2}+\frac{\beta_{0} \hbar^{2}}{2 x_{3} x_{4}}\left(\frac{n_{z} \pi}{d_{z}}\right)^{2}+\frac{1}{m_{1}}\right\}, \\
c_{1}\left(E_{F}, n_{y}, n_{z}\right)= & \left\{E_{F}\left(1+\beta_{0} E_{F}\right)+\beta_{0} E_{F} \frac{\hbar^{2}}{2 x_{5}}\left(\frac{n_{y} \pi}{d_{y}}\right)^{2}+\beta_{0} E_{F} \frac{\hbar^{2}}{2 x_{6}}\left(\frac{n_{z} \pi}{d_{z}}\right)^{2}-\left(1+\beta_{0} E_{F}\right)\left[\frac{\hbar^{2}}{2 x_{2}}\left(\frac{n_{y} \pi}{d_{y}}\right)^{2}\right]-\beta_{0} \frac{\hbar^{4}}{4 x_{2} x_{5}}\left(\frac{n_{y} \pi}{d_{y}}\right)^{4}\right. \\
& -\beta_{0} \frac{\hbar^{4}}{4 x_{2} x_{6}}\left(\frac{n_{y} \pi}{d_{y}}\right)^{2}\left(\frac{n_{z} \pi}{d_{z}}\right)^{2}-\left(1+\beta_{0} E_{F}\right) \frac{\hbar^{2}}{2 x_{3}}\left(\frac{n_{z} \pi}{d_{z}}\right)^{2}-\beta_{0} \frac{\hbar^{4}}{4 x_{3} x_{5}}\left(\frac{n_{y} \pi}{d_{y}}\right)^{2}\left(\frac{n_{z} \pi}{d_{z}}\right)^{2}-\beta_{0} \frac{\hbar^{4}}{4 x_{3} x_{6}}\left(\frac{n_{y} \pi}{d_{y}}\right)^{2}\left(\frac{n_{z} \pi}{d_{z}}\right)^{2} \\
& \left.-\frac{\hbar^{2}}{2 m_{2}}\left(\frac{n_{y} \pi}{d_{y}}\right)^{2}-\frac{\hbar^{2}}{2 m_{3}}\left(\frac{n_{z} \pi}{d_{z}}\right)^{2}\right\},
\end{aligned}
$$

and

$$
T_{8}\left(E_{F}, n_{x}, n_{y}\right) \equiv \sum_{r=1}^{s_{0}} Z_{r} T_{7}\left(E_{F}, n_{x}, n_{y}\right) .
$$

Thus, using Eqs. (5) and (34), the expression of the TPM

$$
G=\left(\frac{\pi^{2} k_{B}^{2} T}{3 e} \sum_{\frac{n_{y}=1}{n_{y_{\max }}}}^{\sum_{n_{y}=1}^{n_{y_{\max }}} \sum_{n_{z}=1}^{n_{z_{\max }}}\left[T_{7}^{\prime}\left(E_{F}, n_{y}, n_{z}\right)+T_{8}^{\prime}\left(E_{F}, n_{y}, n_{z}\right)\right]} .\right.
$$

for QWs of IV-VI compounds can be written as 
Under the conditions $m_{l}^{ \pm} \rightarrow \infty, m_{t}^{ \pm} \rightarrow \infty$, and $m_{l}^{*}=m_{t}^{*}$ $=m^{*}$, Eq. (32) gets simplified into the form as given by Eq. (19).

\section{F. Investigation of TPM for QWs stressed compounds}

The dispersion relation of the conduction electrons in bulk specimens of stressed materials can be written as ${ }^{95}$

$$
\frac{k_{x}^{2}}{\left[a^{*}(E)\right]^{2}}+\frac{k_{y}^{2}}{\left[b^{*}(E)\right]^{2}}+\frac{k_{z}^{2}}{\left[c^{*}(E)\right]^{2}}=1,
$$

where

$$
\begin{aligned}
& {\left[a^{*}(E)\right]^{2}=\frac{k_{0}^{*}(E)}{A_{0}^{*}(E)+\frac{1}{2} D_{0}^{*}(E)},} \\
& k_{0}^{*}(E)=\left(E-C_{1}^{c} \varepsilon-\frac{2 C_{2}^{2} \varepsilon_{x y}^{2}}{3 E_{g}^{\prime}}\right) \frac{3 E_{g}^{\prime}}{2 B_{2}^{* 2}},
\end{aligned}
$$

$\varepsilon$ is the trace of the strain tensor $\hat{\in}$, which can be written as

$$
\hat{\epsilon}=\left|\begin{array}{ccc}
\in_{x x} & \epsilon_{x y} & o \\
\epsilon_{x y} & \epsilon_{y y} & o \\
o & o & \epsilon_{z z}
\end{array}\right|,
$$

$\varepsilon_{x x}, \varepsilon_{x y}, \varepsilon_{y y}$, and $\varepsilon_{z z}$ are its various elements, $C_{1}^{c}$ is the conduction-band deformation potential constant, $C_{2}^{c}$ is a constant that describes the strain interaction between the conduction and the valance bands, $E_{g}^{\prime}=E+\bar{A}, \bar{A}=E_{g}-C_{1}^{c} \varepsilon, B_{2}^{*}$ is the momentum matrix element,

$$
A_{0}^{*}(E)=\left(1-\frac{\left(a_{0}+C_{1}^{c}\right) \varepsilon}{E_{g}^{\prime}}+\frac{3 b_{0} \varepsilon_{x x}}{2 E_{g}^{\prime}}-\frac{b_{0} \varepsilon}{2 E_{g}^{\prime}}\right),
$$

$a_{0}=-\left(x_{0}+2 y_{0}\right) / 3, b_{0}=\left(x_{0}-y_{0}\right) / 3$, where $x_{0}, y_{0}$, and $Z$ are the matrix elements of the strain projection operator,

$$
\begin{aligned}
& D_{0}^{*}(E)=\frac{\sqrt{3} d \varepsilon_{x y}}{E_{g}^{\prime}}, \\
& d=2 Z / \sqrt{3}, \\
& {\left[b^{*}(E)\right]^{2}=\frac{k_{0}^{*}(E)}{\left[A_{0}^{*}(E)-\frac{1}{2} D_{0}^{*}(E)\right]},} \\
& {\left[c^{*}(E)\right]^{2}=\frac{k_{0}^{*}(E)}{L(E)},}
\end{aligned}
$$

and

$$
L(E)=\left(1+\frac{\left(a_{0}+C_{1}^{c}\right) \varepsilon}{E_{g}^{\prime}}+\frac{3 b_{0} \varepsilon_{z z}}{2 E_{g}^{\prime}}-\frac{b_{0} \varepsilon}{2 E_{g}^{\prime}}\right) .
$$

The 1D electron energy spectrum in QWs of stressed materials assumes the form

$$
\frac{k_{x}^{2}}{\left[a^{*}(E)\right]^{2}}+\frac{1}{\left[b^{*}(E)\right]^{2}}\left(n_{y} \pi / d_{y}\right)^{2}+\frac{1}{\left[c^{*}(E)\right]^{2}}\left(n_{z} \pi / d_{z}\right)^{2}=1 .
$$

Using Eq. (37), the electron concentration per unit length can be written as

$$
n_{1 \mathrm{D}}=\left(\frac{2}{\pi}\right) \sum_{n_{y}=1}^{n_{y_{\max }}} \sum_{n_{z=1}}^{n_{z_{\max }}}\left[T_{9}\left(E_{F}, n_{y}, n_{z}\right)+T_{10}\left(E_{F}, n_{y}, n_{z}\right)\right],
$$

where

$$
\begin{aligned}
T_{9}\left(E_{F}, n_{y}, n_{z}\right)= & a^{*}\left(E_{F}\right)\left[1-\left\{\left[b^{*}\left(E_{F}\right)\right]^{-2}\left(n_{y} \pi / d_{y}\right)^{2}\right\}\right. \\
& \left.-\left\{\left[c^{*}\left(E_{F}\right)\right]^{-2}\left(n_{z} \pi / d_{z}\right)^{2}\right\}\right]^{1 / 2}
\end{aligned}
$$

and

$$
T_{10}\left(E_{F}, n_{y}, n_{z}\right) \equiv \sum_{r=1}^{s_{o}} Z_{r} T_{9}\left(E_{F}, n_{y}, n_{z}\right) .
$$

Using Eqs. (5) and (38), the TPM is given by

$$
G=\left(\frac{\pi^{2} k_{B}^{2} T}{3 e}\right) \frac{\sum_{n_{y}=1}^{n_{y_{\max }}} \sum_{n_{z=1}}^{n_{z_{\max }}}\left[T_{9}^{\prime}\left(E_{F}, n_{y}, n_{z}\right)+T_{10}^{\prime}\left(E_{F}, n_{y}, n_{z}\right)\right]}{\sum_{n_{y}=1} \sum_{n_{\text {max }}}^{n_{z_{\max }}}\left[T_{9}\left(E_{F}, n_{y}, n_{z}\right)+T_{10}\left(E_{F}, n_{y}, n_{z}\right)\right]} .
$$

In the absence of stress together with the substitution $B_{2}^{* 2}=3 \hbar^{2}\left(E_{g} / 4 m^{*}\right)$, Eq. (36) assumes the same form as given by Eq. (19).

\section{G. Investigations of TPM for QWs of $n$-GaP}

The dispersion relation of the conduction electrons in bulk specimens of $n-\mathrm{GaP}$ is given by ${ }^{96}$

$$
\begin{aligned}
E= & \frac{\hbar^{2} k_{s}^{2}}{2 m_{t}^{*}}+\frac{\hbar^{2}}{2 m_{l}^{*}}\left(\wp k_{s}^{2}+k_{z}^{2}+k_{0}^{2}\right)-\left(\frac{\hbar^{4} k_{0}^{2}}{m_{l}^{* 2}}\left(k_{s}^{2}+k_{z}^{2}\right)\right. \\
& \left.+\left|V_{G}\right|^{2}\right)^{1 / 2}-\frac{\hbar^{2}}{2 m_{l}^{*}} k_{0}^{2}+\left|V_{G}\right|,
\end{aligned}
$$

where, $k_{0},\left|V_{G}\right|$, and $\wp$ are constants of the energy spectrum.

The 1D dispersion relation in QWs of $n$-GaP can be expressed as

$$
\begin{aligned}
E= & \sigma_{1} k_{s 0}^{2}+C_{2}\left(n_{z} \pi / d_{z}\right)^{2}+\left|V_{G}\right|-\left[D_{1} k_{s 0}^{2}+\left|V_{G}\right|^{2}\right. \\
& \left.+D_{1}\left(n_{z} \pi / d_{z}\right)^{2}\right],
\end{aligned}
$$

where

$$
\sigma_{1}=\left(\frac{\hbar^{2}}{2 m_{t}^{*}}+\frac{\hbar^{2} \wp}{2 m_{l}^{*}}\right),
$$

$k_{s 0}^{2}=k_{x}^{2}+\left(\frac{n_{y} \pi}{d_{y}}\right)^{2}$,

$C_{2}=\frac{\hbar^{2}}{2 m_{l}^{*}}$,

and

$$
D_{1}=\left(\hbar^{4} k_{o}^{2} /\left[m_{l}^{*}\right]^{2}\right) .
$$


Using Eq. (41), the electron concentration per unit length in QWs of $n-\mathrm{GaP}$ assumes the form

$$
n_{1 D}=\left(\frac{2 g_{v}}{\pi \sigma_{1}}\right) \sum_{n_{y}=1}^{n_{y_{\max }}} \sum_{n_{z=1}}^{n_{z_{\max }}}\left[T_{11}\left(E_{F}, n_{y}, n_{z}\right)+T_{12}\left(E_{F}, n_{y}, n_{z}\right)\right],
$$

where

$$
\begin{aligned}
& T_{11}\left(E_{F}, n_{y}, n_{z}\right)=\left[\left(\left\{2 \sigma_{1}\left(E_{F}-t_{1}\right)+D_{1}\right\}-\left\{\left[2 a\left(E_{F}-t_{1}\right)\right.\right.\right.\right. \\
& \left.\left.\left.+D_{1}\right]^{2}-4 \sigma_{1}^{2}\left[\left(E_{F}-t_{1}\right)^{2}-t_{2}\right]\right\}^{1 / 2}\right)\left(2 \sigma_{1}^{2}\right)^{-1} \\
& \left.-\left(\frac{n_{y} \pi}{d_{y}}\right)^{2}\right]^{1 / 2} \text {, } \\
& t_{1}=\left|V_{G}\right|+C_{2}\left(n_{z} \pi / d_{z}\right)^{2}, \\
& t_{2}=\left[\left|V_{G}\right|^{2}+D_{1}\left(n_{z} \pi / d_{z}\right)^{2}\right],
\end{aligned}
$$

and

$$
T_{12}\left(E_{F}, n_{y}, n_{z}\right)=\sum_{r=1}^{S_{o}} Z_{r}\left[T_{11}\left(E_{F}, n_{y}, n_{z}\right)\right] .
$$

The use of Eqs. (5) and (42) leads to the expression of the TPM for QWs of $n$-GaP as

$$
G=\left(\frac{\pi^{2} k_{B}^{2} T}{3 e}\right) \sum_{n_{y}=1}^{\sum_{n_{y}=1}^{n_{y_{\max }}} \sum_{n_{z=1}}^{n_{y_{\max }}} \sum_{n_{z=1}}^{n_{z_{\max }}}\left[T_{11}^{\prime}\left(E_{F}, n_{y}, n_{z}\right)+T_{12}^{\prime}\left(E_{F}, n_{y}, n_{z}\right)\right]} .
$$

Under the conditions $k_{0}=0,\left|V_{G}\right|=0, m_{t}^{*}=m_{l}^{*}=m^{*}$, and $\wp=0$, Eq. (40) assumes the form as given by Eq. (22).

\section{H. Investigation of the TPM for the QWs of p-type platinum antimonide $\left(\boldsymbol{p}-\mathrm{PtSb}_{2}\right)$}

The dispersion relation of the carriers in $p$-type $\mathrm{PtSb}_{2}$ can be written as ${ }^{110}$

$$
\begin{aligned}
(E & \left.+\lambda_{1} \frac{a^{2}}{4} k^{2}-l_{1} k_{s}^{2} \frac{a^{2}}{4}\right)\left(E+\delta_{0}^{\prime}-v_{1} \frac{a^{2}}{4} k^{2}-n^{\prime} \frac{a^{2}}{4} k_{s}^{2}\right) \\
= & I_{0} \frac{a^{4}}{16} k^{4},
\end{aligned}
$$

where $\lambda_{1}, l_{1}, \delta_{0}^{\prime}, \nu_{1}, n^{\prime}$, and $I_{0}$ are the energy band constants and $a$ is the lattice constant as defined in Ref. 110.

Inserting the negative signs of $l_{1}$ and $n^{\prime}$, we get

$$
\left(E+\omega_{1}^{\prime} k_{s}^{2}+\omega_{2}^{\prime} k_{z}^{2}\right)\left(E+\delta_{0}^{\prime}+\omega_{3}^{\prime} k_{s}^{2}-\omega_{4}^{\prime} k_{z}^{2}\right)=I_{1}\left(k_{z}^{2}+k_{s}^{2}\right)^{2},
$$

where

$$
\omega_{1}^{\prime}=\left(\lambda_{1} \frac{a^{2}}{4}-l_{1} \frac{a^{2}}{4}\right),
$$

$$
\begin{aligned}
& \omega_{2}^{\prime}=\lambda_{1} \frac{a^{2}}{4}, \\
& \omega_{3}^{\prime}=\left(n^{\prime} \frac{a^{2}}{4}-v_{1} \frac{a^{2}}{4}\right), \\
& \omega_{4}^{\prime}=n^{\prime} \frac{a^{2}}{4}, \\
& I_{1}=I_{0}\left(\frac{a}{4}\right)^{2} .
\end{aligned}
$$

The use of Eq. (45) leads to the expression of the 1D dispersion law in QWs of $p-\mathrm{PtSb}_{2}$ as

$$
\begin{aligned}
k_{x}^{2}+\left(\frac{n_{y} \pi}{d_{y}}\right)^{2}= & {\left[2 N_{1}\right]^{-1}\left[N_{2}\left(E, n_{z}\right)\right.} \\
& \left.+\sqrt{N_{2}^{2}\left(E, n_{z}\right)+4 N_{1} N_{3}\left(E, n_{z}\right)}\right],
\end{aligned}
$$

where

$$
\begin{aligned}
& N_{1}=\left(I_{1}-\omega_{1}^{\prime} \omega_{3}^{\prime}\right), \\
& N_{2}\left(E, n_{z}\right)= {\left[\omega_{3}^{\prime} E-\omega_{1}^{\prime}\left\{E+\delta_{0}^{\prime}-\omega_{4}^{\prime}\left(\frac{n_{z} \pi}{d_{z}}\right)^{2}\right.\right.} \\
&\left.\left.+\omega_{2}^{\prime} \omega_{3}^{\prime}\left(\frac{n_{z} \pi}{d_{z}}\right)^{2}-2 I_{1}\left(\frac{n_{z} \pi}{d_{z}}\right)^{2}\right\}\right], \\
& N_{3}\left(E, n_{z}\right)=\left\{E\left[E+\delta_{0}^{\prime}-\omega_{4}^{\prime}\left(\frac{n_{z} \pi}{d_{z}}\right)^{2}\right]+\omega_{2}^{\prime}\left(\frac{n_{z} \pi}{d_{z}}\right)^{2}\right. \\
&\left.\times\left[E+\delta_{0}^{\prime}-\omega_{4}^{\prime}\left(\frac{n_{z} \pi}{d_{z}}\right)^{2}\right]-I_{1}\left(\frac{n_{z} \pi}{d_{z}}\right)^{4}\right\} .
\end{aligned}
$$

The formulation of the TPM requires the expression of 1D carrier concentration per unit length, which can, in turn, be written as

$$
n_{1 \mathrm{D}}=\left(\frac{2 g_{v}}{\pi}\right) \sum_{n_{y}=1}^{n_{y_{\max }}} \sum_{n_{z=1}}^{n_{z_{\max }}}\left[T_{13}\left(E_{F}, n_{y}, n_{z}\right)+T_{14}\left(E_{F}, n_{y}, n_{z}\right)\right],
$$

where

$$
\begin{aligned}
& T_{13}\left(E_{F}, n_{y}, n_{z}\right)= {\left[\Omega_{4}\left(E_{F}, n_{z}\right)-\left(\frac{n_{y} \pi}{d_{y}}\right)^{2}\right]^{1 / 2}, } \\
& \Omega_{4}\left(E_{F}, n_{z}\right)= {\left[N_{2}\left(E_{F}, n_{z}\right)+\sqrt{N_{2}^{2}\left(E_{F}, n_{z}\right)+4 N_{1} N_{3}\left(E_{F}, n_{z}\right)}\right] } \\
& \times\left(2 N_{1}\right)^{-1},
\end{aligned}
$$

and

$$
T_{14}\left(E_{F}, n_{y}, n_{z}\right)=\sum_{r=1}^{S} Z_{r}\left[T_{13}\left(E_{F}, n_{y}, n_{z}\right)\right] .
$$

The use of Eqs. (5) and (47) leads to the expression of the TPM in this case as 


$$
G=\left(\frac{\pi^{2} k_{B}^{2} T}{3 e} \sum_{\frac{n_{y}=1}{n_{y_{\max }}} \sum_{n_{z=1}}^{n_{y_{\max }}} \sum_{n_{y}=1}^{n_{z_{\max }}}\left[T_{n_{z=1}}^{\prime}\left(E_{F}, n_{y}, n_{z}\right)+T_{14}^{\prime}\left(E_{F}, n_{y}, n_{z}\right)\right]}^{\left.\left.n_{F}, n_{y}, n_{z}\right)+T_{14}\left(E_{F}, n_{y}, n_{z}\right)\right]} .\right.
$$

\section{Investigation of the TPM for the QWs of n-GaSb}

The dispersion relation of the conduction-band electrons in bulk specimens of $n$-GaSb can be written as ${ }^{111}$

$$
\begin{aligned}
E= & \left(-\frac{E_{g}}{2}+\frac{E_{g}}{2}\left(1+\alpha_{0} k^{2}\right)^{1 / 2}+\frac{\zeta_{0} \hbar^{2} k^{2}}{2 m_{o}}\right. \\
& \left.+\frac{v_{0} f_{1}(k) \hbar^{2}}{2 m_{o}} \pm \frac{\omega_{0} f_{2}(k) \hbar^{2}}{2 m_{o}}\right),
\end{aligned}
$$

where $\alpha_{0}=4 P^{2}\left(E_{g}+\frac{2}{3} \Delta\right)\left[E_{g}^{2}\left(E_{g}+\Delta\right)\right]^{-1}, P$ is the momentum matrix element, $f_{1}(k)=k^{-2}\left(k_{x}^{2} k_{y}^{2}+k_{y}^{2} k_{z}^{2}+k_{z}^{2} k_{x}^{2}\right)$ represents the warping of the Fermi surface, $f_{2}(k)=\left[\left\{k^{2}\left(k_{x}^{2} k_{y}^{2}+k_{y}^{2} k_{z}^{2}+k_{z}^{2} k_{x}^{2}\right)\right.\right.$ $\left.\left.-9 k_{x}^{2} k_{y}^{2} k_{z}^{2}\right\}^{1 / 2} k^{-1}\right]$ represents the inversion asymmetry splitting of the conduction band, and $\zeta_{0}, v_{0}$, and $\omega_{0}$ represent the constants of the electron spectrum in this case.

The use of the method of the spherical averaging ${ }^{112}$ leads to the simplified expression of the above-mentioned dispersion relation as

$$
k^{2}=A_{5}(E),
$$

in which

$$
\begin{aligned}
A_{5}(E)= & \left(2 S_{1}^{2}\right)^{-1}\left\{\left[S_{1}\left(1+2 \beta_{0} E\right)-\alpha_{0}\right]-\left[\alpha_{0}^{2}-4 \alpha_{0} S_{1}(1\right.\right. \\
& \left.\left.\left.+2 \beta_{0} E\right)+4 S_{1}^{2}\left(1-20 \beta_{0} E-28 \beta_{0}^{2} E^{2}\right)\right]^{1 / 2}\right\},
\end{aligned}
$$

where

$$
S_{1}=\left(\frac{\beta_{0} \hbar^{2}}{m_{0}}\right)\left(\zeta_{0}+0.1 \nu_{0}+0.856 \omega_{0}\right) .
$$

Using Eq. (50), the 1D dispersion relation in QWs of $n$-GaSb can be written as

$$
k_{x}^{2}=A_{5}(E)-\left(\frac{n_{y} \pi}{d_{y}}\right)^{2}-\left(\frac{n_{z} \pi}{d_{z}}\right)^{2} .
$$

The expression of electron concentration per unit length in QWs of $n-\mathrm{GaSb}$ can, in turn, be written as

$$
n_{1 \mathrm{D}}=\left(\frac{2}{\pi}\right) \sum_{n_{y}=1}^{n_{y_{\max }}} \sum_{n_{z=1}}^{n_{z_{\max }}}\left[T_{15}\left(E_{F}, n_{y}, n_{z}\right)+T_{16}\left(E_{F}, n_{y}, n_{z}\right)\right],
$$

where

$$
T_{15}\left(E_{F}, n_{y}, n_{z}\right)=\left[A_{5}\left(E_{F}\right)-\left(\frac{n_{y} \pi}{d_{y}}\right)^{2}-\left(\frac{n_{z} \pi}{d_{z}}\right)^{2}\right]^{1 / 2}
$$

and

$$
T_{16}\left(E_{F}, n_{y}, n_{z}\right)=\sum_{r=1}^{s_{o}} Z_{r}\left[T_{15}\left(E_{F}, n_{y}, n_{z}\right)\right] .
$$

The use of Eqs. (5) and (52) leads to the expression of the TPM in this case as

$$
G=\left(\frac{\pi^{2} k_{B}^{2} T}{3 e}\right) \frac{\sum_{n_{y}=1}^{n_{y_{\max }}} \sum_{n_{z=1}}^{n_{z_{\max }}}\left[T_{15}^{\prime}\left(E_{F}, n_{y}, n_{z}\right)+T_{16}^{\prime}\left(E_{F}, n_{y}, n_{z}\right)\right]}{\sum_{n_{y}=1}^{n_{y_{\max }}} \sum_{n_{z=1}}^{n_{\max }}\left[T_{15}\left(E_{F}, n_{y}, n_{z}\right)+T_{16}\left(E_{F}, n_{y}, n_{z}\right)\right]} .
$$

Under the substitutions $\zeta_{0}=0, v_{0}=0, \omega_{0}=0$, and

$$
P^{2}=\frac{\hbar^{2}}{2 m^{*}} \frac{E_{g}\left(E_{g}+\Delta\right)}{\left(E_{g}+\frac{2}{3} \Delta\right)},
$$

Eq. (49) gets simplified into that given by Eq. (19).

\section{J. Investigation of the TPM for the QWs of Bi in accordance with the McClure and Choi, the Hybrid, the Cohen, the Lax, and the parabolic ellipsoidal models}

\section{The McClure and Choi model}

The dispersion relation of the carriers in Bi can be written, following McClure and Choi, ${ }^{107}$ as

$$
\begin{aligned}
E\left(1+\beta_{0} E\right)= & \frac{p_{x}^{2}}{2 m_{1}}+\frac{p_{y}^{2}}{2 m_{2}}+\frac{p_{z}^{2}}{2 m_{3}}+\frac{p_{y}^{2}}{2 m_{2}} \beta_{0} E\left\{1-\left(\frac{m_{2}}{m_{2}^{\prime}}\right)\right\} \\
& +\frac{p_{y}^{4} \beta_{0}}{4 m_{2} m_{2}^{\prime}}-\frac{\beta_{0} p_{x}^{2} p_{y}^{2}}{4 m_{1} m_{2}}-\frac{\beta_{0} p_{y}^{2} p_{z}^{2}}{4 m_{2} m_{3}}
\end{aligned}
$$

where $p_{i}=\hbar k_{i} ; i=x, y$, and $z ; m_{1}, m_{2}$, and $m_{3}$ are the effective carrier masses at the band edge along the $x, y$, and $z$ directions, respectively; and $m_{2}^{\prime}$ is the effective-mass tensor component at the top of the valence band (for electrons) or at the bottom of the conduction band (for holes).

The 1D dispersion relation in QWs of Bi can be expressed as

$$
\begin{aligned}
k_{x}^{2}\left[\frac{\hbar^{2}}{2 m_{1}}-\left(\frac{\beta_{0} \hbar^{2}}{4 m_{1} m_{2}}\left(\frac{n_{y} \pi}{d_{y}}\right)^{2}\right)\right] \\
+\left(\frac{n_{z} \pi}{d_{z}}\right)^{2}\left[\frac{\hbar^{2}}{2 m_{3}}-\left(\frac{\beta_{0} \hbar^{2}}{4 m_{2} m_{3}}\left(\frac{n_{y} \pi}{d_{y}}\right)^{2}\right)\right] \\
=E\left(1+\beta_{0} E\right)-\frac{\beta_{0}}{4 m_{2} m_{2}^{\prime}}\left(\frac{\hbar n_{y} \pi}{d_{y}}\right)^{4}-\beta_{0} E\left\{1-\left(\frac{m_{2}}{m_{2}^{\prime}}\right)\right\} \\
\quad \times\left(\frac{\hbar n_{y} \pi}{d_{y}}\right)^{2} .
\end{aligned}
$$

Using Eq. (55), the electron concentration per unit length for QWs of Bi can be written as

$$
n_{1 \mathrm{D}}=\left(\frac{2 g_{v}}{\pi}\right) \sum_{n_{y}=1}^{n_{y_{\max }}} \sum_{n_{z=1}}^{n_{z_{\max }}}\left[T_{17}\left(E_{F}, n_{y}, n_{z}\right)+T_{18}\left(E_{F}, n_{y}, n_{z}\right)\right],
$$




$$
\begin{aligned}
T_{17}\left(E_{F}, n_{y}, n_{z}\right) & \left\{\left[E_{F}\left(1+\beta_{0} E_{F}\right)-\frac{\beta_{0}}{4 m_{2} m_{2}^{\prime}}\left(\frac{\hbar n_{y} \pi}{d_{y}}\right)^{4}\right.\right. \\
& -\beta_{0} E_{F}\left\{1-\left(\frac{m_{2}}{m_{2}^{\prime}}\right)\right\}\left(\frac{\hbar n_{y} \pi}{d_{y}}\right)^{2} \\
& \left.\left.-\left(\frac{n_{z} \pi}{d_{z}}\right)^{2}\left[\frac{\hbar^{2}}{2 m_{3}}-\left(\frac{\beta_{0} \hbar^{2}}{4 m_{2} m_{3}}\left(\frac{n_{y} \pi}{d_{y}}\right)^{2}\right)\right]\right]\right\}^{1 / 2} \\
& \times\left[\frac{\hbar^{2}}{2 m_{1}}-\frac{\beta_{0} \hbar^{2}}{4 m_{1} m_{2}}\left(\frac{n_{y} \pi}{d_{y}}\right)^{2}\right]^{-1 / 2}
\end{aligned}
$$

and

$$
T_{18}\left(E_{F}, n_{y}, n_{z}\right)=\sum_{r=1}^{s_{o}} Z_{r}\left[T_{17}\left(E_{F}, n_{y}, n_{z}\right)\right] .
$$

Using Eqs. (5) and (56), the expression of the TPM in this case can be written as

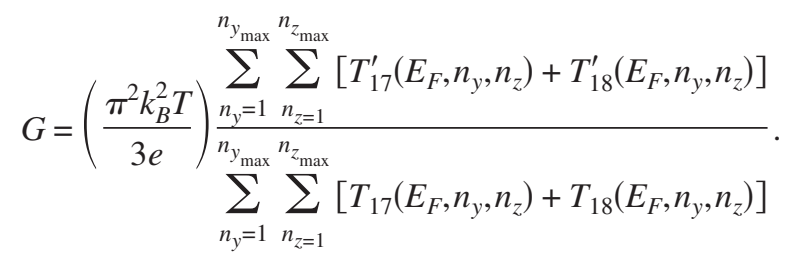

Under the condition $\beta_{0} \rightarrow 0$ and $m_{1}=m_{2}=m_{3}=m^{*}$, Eq. (54) gets simplified to Eq. (22).

\section{The Hybrid model}

The electron dispersion law in bulk specimens of $\mathrm{Bi}$ in accordance with the Hybrid model ${ }^{106}$ can be represented as

$$
E\left(1+\beta_{0} E\right)=\frac{\theta_{0}(E) \hbar^{2} k_{y}^{2}}{2 M_{2}}+\frac{\beta_{0} \gamma_{0} \hbar^{4} k_{y}^{4}}{4 M_{2}^{2}}+\frac{\hbar^{2} k_{x}^{2}}{2 m_{1}}+\frac{\hbar^{2} k_{z}^{2}}{2 m_{3}},
$$

in which

$$
\begin{aligned}
& \theta_{0}(E)=\left[1+\beta_{0} E\left(1-\gamma_{0}\right)+\delta_{0}\right], \\
& \gamma_{0}=\frac{M_{2}}{m_{2}}, \\
& \delta_{0}=\frac{M_{2}}{M_{2}^{\prime}},
\end{aligned}
$$

and the other notations are defined in Ref. 106.

The 1D electron dispersion relation assumes the form

$$
\begin{aligned}
\frac{\hbar^{2} k_{x}^{2}}{2 m_{1}}+\frac{\hbar^{2}}{2 m_{3}}\left(\frac{n_{z} \pi}{d_{z}}\right)^{2}= & E\left(1+\beta_{0} E\right)-\frac{\theta_{0}(E) \hbar^{2}}{2 M_{2}}\left(\frac{n_{y} \pi}{d_{y}}\right)^{2} \\
& -\left(\frac{\beta_{0} \gamma_{0} \hbar^{4}}{4 M_{2}^{2}}\left(\frac{n_{y} \pi}{d_{y}}\right)^{4}\right) .
\end{aligned}
$$

The use of Eq. (59) leads to the expression of the electron concentration per unit length in QWs for $\mathrm{Bi}$ in this case as

$$
\begin{aligned}
n_{1 \mathrm{D}}= & \left(\frac{2 g_{v}}{\pi} \frac{\sqrt{2 m_{1}}}{\hbar}\right) \sum_{n_{y}=1}^{n_{y_{\max }}} \sum_{n_{z=1}}^{n_{\max }}\left[T_{19}\left(E_{F}, n_{y}, n_{z}\right)\right. \\
& \left.+T_{20}\left(E_{F}, n_{y}, n_{z}\right)\right]
\end{aligned}
$$

in which

$$
\begin{aligned}
T_{19}\left(E_{F}, n_{y}, n_{z}\right)= & {\left[E_{F}\left(1+\beta_{0} E_{F}\right)-\frac{\theta_{0}(E) \hbar^{2}}{2 M_{2}}\left(\frac{n_{y} \pi}{d_{y}}\right)^{2}\right.} \\
& \left.-\frac{\beta_{0} \gamma_{0}}{4 M_{2}^{2}} \hbar^{4}\left(\frac{n_{y} \pi}{d_{y}}\right)^{4}-\frac{\hbar^{2}}{2 m_{3}}\left(\frac{n_{z} \pi}{d_{z}}\right)^{2}\right]^{1 / 2}
\end{aligned}
$$

and

$$
T_{20}\left(E_{F}, n_{y}, n_{z}\right)=\sum_{r=1}^{s_{o}} Z_{r}\left[T_{19}\left(E_{F}, n_{y}, n_{z}\right)\right] .
$$

Using Eqs. (5) and (60), the expression of the TPM in this case assumes the form

$$
G=\left(\frac{\pi^{2} k_{B}^{2} T}{3 e} \sum_{\frac{n_{y}=1}{n_{y_{\max }}} \sum_{n_{z=1}}^{n_{y_{\max }}} \sum_{n_{y}=1}^{n_{z_{\max }}}\left[T_{19}^{\prime}\left(E_{F}, n_{y}, n_{z}\right)+T_{20}^{\prime}\left(E_{F}, n_{y}, n_{z}\right)\right]}^{\left.n_{z=1}\left(E_{F}, n_{y}, n_{z}\right)+T_{20}\left(E_{F}, n_{y}, n_{z}\right)\right]} .\right.
$$

\section{The Cohen model}

In accordance with the Cohen model, ${ }^{105}$ the dispersion law of the carriers in $\mathrm{Bi}$ is given by

$$
\begin{aligned}
E\left(1+\beta_{0} E\right)= & \frac{p_{x}^{2}}{2 m_{1}}+\frac{p_{z}^{2}}{2 m_{3}}-\frac{\beta_{0} E p_{y}^{2}}{2 m_{2}^{\prime}}+\frac{p_{y}^{2}\left(1+\beta_{0} E\right)}{2 m_{2}} \\
& +\frac{\beta_{0} p_{y}^{4}}{4 m_{2} m_{2}^{\prime}} .
\end{aligned}
$$

The 1D dispersion relation in QWs for Bi can be expressed as

$$
\begin{gathered}
E\left(1+\beta_{0} E\right)+\frac{\beta_{0} E \hbar^{2}}{2 m_{2}^{\prime}}\left(\frac{n_{y} \pi}{d_{y}}\right)^{2}-\frac{\left(1+\beta_{0} E\right) \hbar^{2}}{2 m_{2}}\left(\frac{n_{y} \pi}{d_{y}}\right)^{2} \\
-\frac{\beta_{0} \hbar^{4}}{4 m_{2} m_{2}^{\prime}}\left(\frac{n_{y} \pi}{d_{y}}\right)^{4}-\frac{\hbar^{2}}{2 m_{3}}\left(\frac{n_{z} \pi}{d_{z}}\right)^{2}=\frac{\hbar^{2} k_{x}^{2}}{2 m_{1}} .
\end{gathered}
$$

The use of Eq. (63) leads to the expression of the 1D carrier statistics of QWs for $\mathrm{Bi}$ in this case as

$$
\begin{aligned}
n_{1 D}= & \left(\frac{2 g_{v}}{\pi} \frac{\sqrt{2 m_{1}}}{\hbar}\right) \sum_{n_{y}=1}^{n_{y_{\max }}} \sum_{n_{z=1}}^{n_{z_{\max }}}\left[T_{21}\left(E_{F}, n_{y}, n_{z}\right)\right. \\
& \left.+T_{22}\left(E_{F}, n_{y}, n_{z}\right)\right]
\end{aligned}
$$

in which 


$$
\begin{aligned}
T_{21}\left(E_{F}, n_{y}, n_{z}\right)= & {\left[E_{F}\left(1+\beta_{0} E_{F}\right)+\frac{\beta_{0} E_{F} \hbar^{2}}{2 m_{2}^{\prime}}\left(\frac{n_{y} \pi}{d_{y}}\right)^{2}\right.} \\
& -\left(\frac{\left(1+\beta_{0} E_{F}\right) \hbar^{2}}{2 m_{2}}\right) \\
& \times\left(\frac{n_{y} \pi}{d_{y}}\right)^{2}-\left(\frac{\beta_{0} \hbar^{4}}{4 m_{2} m_{2}^{\prime}}\left(\frac{n_{y} \pi}{d_{y}}\right)^{4}\right) \\
& \left.-\left[\frac{\hbar^{2}}{2 m_{3}}\left(\frac{n_{z} \pi}{d_{z}}\right)^{2}\right]\right]^{1 / 2}
\end{aligned}
$$

and

$$
T_{22}\left(E_{F}, n_{y}, n_{z}\right)=\sum_{r=1}^{s_{o}} Z_{r}\left[T_{21}\left(E_{F}, n_{y}, n_{z}\right)\right] .
$$

The use of Eqs. (5) and (64) leads to the expression of the TPM of QWs of Bi as

$$
G=\left(\frac{\pi^{2} k_{B}^{2} T}{3 e}\right) \frac{\sum_{n_{y}=1}^{n_{y_{\max }}} \sum_{n_{z=1}}^{n_{z_{\max }}}\left[T_{21}^{\prime}\left(E_{F}, n_{y}, n_{z}\right)+T_{22}^{\prime}\left(E_{F}, n_{y}, n_{z}\right)\right]}{\sum_{n_{\max }=1}} \sum_{n_{z=1}}^{n_{z_{\max }}}\left[T_{21}\left(E_{F}, n_{y}, n_{z}\right)+T_{22}\left(E_{F}, n_{y}, n_{z}\right)\right] .
$$

\section{The Lax model}

The electron energy spectra in bulk specimens of $\mathrm{Bi}$ in accordance with the Lax model can be written as ${ }^{99}$

$$
E\left(1+\beta_{0} E\right)=\frac{p_{x}^{2}}{2 m_{1}}+\frac{p_{y}^{2}}{2 m_{2}}+\frac{p_{z}^{2}}{2 m_{3}} .
$$

The 1D electron dispersion law is given by

$$
E\left(1+\beta_{0} E\right)-\frac{\hbar^{2}}{2 m_{2}}\left(\frac{n_{y} \pi}{d_{y}}\right)^{2}-\frac{\hbar^{2}}{2 m_{3}}\left(\frac{n_{z} \pi}{d_{z}}\right)^{2}=\frac{\hbar^{2} k_{x}^{2}}{2 m_{1}} .
$$

The electron concentration per unit length can be written as

$$
\begin{aligned}
n_{1 \mathrm{D}}= & \left(\frac{2 g_{v}}{\pi} \frac{\sqrt{2 m_{1}}}{\hbar}\right) \sum_{n_{y}=1}^{n_{y_{\max }}} \sum_{n_{z=1}}^{n_{z_{\max }}}\left[T_{23}\left(E_{F}, n_{y}, n_{z}\right)\right. \\
& \left.+T_{24}\left(E_{F}, n_{y}, n_{z}\right)\right],
\end{aligned}
$$

in which

$$
\begin{aligned}
T_{23}\left(E_{F}, n_{y}, n_{z}\right) \\
=\left[E_{F}\left(1+\beta_{0} E_{F}\right)-\left(\frac{\hbar^{2}}{2 m_{2}}\left(\frac{n_{y} \pi}{d_{y}}\right)^{2}\right)\right. \\
\left.-\left(\frac{\hbar^{2}}{2 m_{3}}\left(\frac{n_{z} \pi}{d_{z}}\right)^{2}\right)\right]^{1 / 2}
\end{aligned}
$$

and

$$
T_{24}\left(E_{F}, n_{y}, n_{z}\right)=\sum_{r=1}^{s_{o}} Z_{r}\left[T_{23}\left(E_{F}, n_{y}, n_{z}\right)\right]
$$

The use of Eqs. (5) and (68) leads to the expression of the TPM of QWs of $\mathrm{Bi}$ as

$$
G=\left(\frac{\pi^{2} k_{B}^{2} T}{3 e}\right)_{\sum_{n_{y}=1}}^{\sum_{n_{y_{\max }}}^{n_{y_{\max }}} \sum_{n_{z=1}}^{n_{z_{\max }}}\left[T_{23}^{\prime}\left(E_{F}, n_{y}, n_{z}\right)+T_{24}^{\prime}\left(E_{F}, n_{y}, n_{z}\right)\right]} .
$$

\section{The parabolic ellipsoidal model}

This type of band model in bulk specimens of $\mathrm{Bi}$ assumes the form ${ }^{97,98}$

$$
E=\frac{p_{x}^{2}}{2 m_{1}}+\frac{p_{y}^{2}}{2 m_{2}}+\frac{p_{z}^{2}}{2 m_{3}} .
$$

The analytical forms of the electron statistics and the TPM in this case are given by Eqs. (23) and (24), where $m^{*}$ should be replaced by $m_{1}$,

$$
\sum_{n_{x}=1}^{n_{x_{\max }}} \sum_{n_{y=1}}^{n_{y_{\max }}}
$$

by

$$
\sum_{n_{y}=1}^{n_{y_{\max }}} \sum_{n_{z=1}}^{n_{z_{\max }}}
$$

and $\eta_{1}$ by

$$
\left(k_{B} T\right)^{-1}\left\{E_{F}-\left[\frac{\hbar^{2}}{2 m_{2}}\left(\frac{n_{y} \pi}{d_{y}}\right)^{2}+\frac{\hbar^{2}}{2 m_{3}}\left(\frac{n_{z} \pi}{d_{z}}\right)^{2}\right]\right\} .
$$

\section{$K$. The results of this paper find the following two important applications in the areas of quantum effect devices}

(i) It is well known that the Einstein relation for the diffusivity-mobility ratio $(D / \mu)$ is an important quantity for studying the transport properties of semiconductor devices since the diffusion constant (a quantity very useful for device analysis but whose exact experimental determination is rather difficult) can be derived from this ratio if one knows the experimental values of the mobility. ${ }^{113}$ The performance of the electronic devices at device terminal and the speed of operation of modern switching transistors are significantly influenced by the degree of carrier degeneracy present in these devices. The simplest way of analyzing such devices taking into account the degeneracy of bands is to use the appropriate Einstein relation to express the performance at the device terminals and switching speed in terms of carrier concentration. ${ }^{114}$ The Einstein relation can, in general, be written as ${ }^{115}$

$$
\frac{D}{\mu}=\left(n_{0} / e\right) / \frac{\partial n_{0}}{\partial E_{F}},
$$

where $n_{0}$ is the electron concentration.

Using Eqs. (71) and (5), we get 


$$
\frac{D}{\mu}=\pi^{2} k_{B} T /\left(3 e^{2} G\right)
$$

Therefore, we can experimentally determine the $D / \mu$ ratio for any materials having arbitrary dispersion laws by knowing the experimental values of $G$. Since $G$ decreases with increasing $n_{0}$, from Eq. (72), we can infer that the $D / \mu$ ratio will increase with an increase in $n_{0}$, and this compatibility is the direct test of our suggestion for an experimental determination of the Einstein relation.

(ii) The knowledge of the carrier contribution to the elastic constants $\left(\Delta C_{44}\right.$ and $\left.\Delta C_{456}\right)$ is very important in studying the mechanical properties of the materials in nanotechnology, and has been investigated extensively in the literature. ${ }^{116}$ The electronic contribution to the second- and third-order elastic constants can be written as ${ }^{116}$

$$
\Delta C_{44}=-\frac{G_{0}^{2}}{9} \frac{\partial n_{0}}{\partial E_{F}}
$$

and

$$
\Delta C_{456}=\frac{G_{0}^{3}}{27} \frac{\partial^{2} n_{0}}{\partial E_{F}^{2}},
$$

where $G_{0}$ is the deformation potential constant. Thus, using Eqs. (5), (73), and (74), we can write

$$
\Delta C_{44}=\left[-n_{0} G_{0}^{2} e G /\left(3 \pi^{2} k_{B}^{2} T\right)\right]
$$

and

Therefore, the experimental graph of $G$ versus $n_{0}$ allows us to determine the electronic contribution to the elastic constants for materials having arbitrary carrier energy spectra.

Thus, we can summarize the whole mathematical background in the following way:

In this article, we have investigated the TPM in CNTs. We have also studied the TPM in QWs of nonlinear optical materials on the basis of a newly formulated electron dispersion law considering the anisotropies of the effective electron masses, the spin-orbit splitting constants, and the influence of crystal-field splitting within the framework of the $\mathbf{k} \cdot \mathbf{p}$ formalism. The corresponding results for the QWs of III-V, ternary, and quaternary compounds form a special case of our generalized analysis. The TPM has also been studied for QWs of II-VI, IV-VI, stressed materials, $n$-GaP, $p$ - $\mathrm{PtSb}_{2}$, and $n$-GaSb compounds by using the models of Hopfield, ${ }^{93}$ Dimmock, ${ }^{94}$ Seiler, ${ }^{95}$ Rees, ${ }^{96}$ Emtage, ${ }^{110}$ and Seiler, ${ }^{111}$ respectively. The TPM for the QWs of bismuth has further been investigated in accordance with the McClure and Choi model, ${ }^{107}$ the Hybrid model, ${ }^{106}$ the Cohen model, ${ }^{105}$ the Lax model,${ }^{99}$ and the parabolic ellipsoidal model,${ }^{98}$ respectively. The well-known expression of the TPM of wide gap materials has been obtained as a special case of our generalized analysis under certain limiting conditions from all the results. This indirect test not only exhibits the mathematical compatibility of our formulation, but it also indicates the fact that our simple analysis is a more generalized one, since one can obtain the corresponding results for bulk specimens of relatively wide gap materials having parabolic energy bands under certain limiting conditions from our present investiga-
TABLE I. The numerical values of the energy band constants of the materials as used in this article.

(a) $n-\mathrm{Cd}_{3} \mathrm{As}_{2}:{ }^{118}\left|E_{g}\right|=0.095 \mathrm{eV}, \Delta_{\|}=0.27 \mathrm{eV}, \Delta_{\perp}=0.25 \mathrm{eV}$,

$m_{\|}^{*}=0.00697 m_{0} m_{\perp}=0.013933 m_{0}$, and $\delta=0.085 \mathrm{eV}$

(b) $n$-CdGeAs $2:{ }^{118} E_{g}=0.57 \mathrm{eV}, \Delta_{\|}=0.30 \mathrm{eV}, \Delta_{\perp}=0.36 \mathrm{eV}$, $m_{\|}^{*}=0.034 m_{0}, m_{\perp}^{*}=0.039 m_{0}$, and $\delta=-0.21 \mathrm{eV}$

(c) $n$-InAs: ${ }^{109} E_{g}=0.4180 \mathrm{eV}, \Delta=0.38 \mathrm{eV}$, and $m^{*}=0.0265 m_{0}$

(d) $n$-InSb: ${ }^{109} E_{g}=0.2352 \mathrm{eV}, \Delta=0.81 \mathrm{eV}$, and $m^{*}=0.01359 m_{0}$

(e) $n$-GaAs: ${ }^{109} E_{g}=1.55 \mathrm{eV}, \Delta=0.35 \mathrm{eV}$, and $m^{*}=0.07 m_{0}$

(f) $\mathrm{Hg}_{1-x} \mathrm{Cd}_{x} \mathrm{Te}^{86}{ }^{86} E_{g}=\left[-0.302+1.93 x+5.35 \times 10^{-4}(1-2 x) T-0.810 x^{2}\right.$

$\left.+0.832 x^{3}\right] \mathrm{eV}, \Delta=\left(0.63+0.24 x-0.27 x^{2}\right) \mathrm{eV}$, and $m^{*}=0.1 m_{0} E_{g}(\mathrm{eV})^{-1}$

(g) $\mathrm{In}_{1-x} \mathrm{Ga}_{x} \mathrm{As}_{y} \mathrm{P}_{1-y} \cdot{ }^{.87} E_{g}=\left(1.337-0.73 y+0.13 y^{2}\right) \mathrm{eV}, \Delta=(0.114+0.26 y$

$\left.-0.22 y^{2}\right) \mathrm{eV}$, and $m^{*}=(0.08-0.039 y) m_{0}$

(h) $\mathrm{CdS}:{ }^{93} m_{\|}^{*}=0.7 m_{0}, m_{\perp}^{*}=1.5 m_{0}$, and $\bar{\lambda}_{0}=1.4 \times 10^{-10} \mathrm{eV} \mathrm{m}$

(i) $n$-PbTe: ${ }^{118} m_{t}^{-}=0.070 m_{0}, m_{l}^{-}=0.54 m_{0}, m_{t}^{+}=0.010 m_{0}, m_{l}^{+}=1.4 m_{0}, P_{\|}$

$=141 \mathrm{meV} \mathrm{nm}, P_{\perp}=486 \mathrm{meV} \mathrm{nm}, g_{v}=4$, and $E_{g}=190 \mathrm{meV}$

(j) $n$-PbSnTe: ${ }^{118} m_{t}^{-}=0.063 m_{0}, m_{l}^{-}=0.41 m_{0}, m_{t}^{+}=0.089 m_{0}, m_{l}^{+}=1.6 m_{0}, P_{\|}$

$=137 \mathrm{meV} \mathrm{nm}, P_{\perp}=464 \mathrm{meV} \mathrm{nm}, g_{v}=4$, and $E_{g}=90 \mathrm{meV}$

(k) $n-\mathrm{Pb}_{1-x} \mathrm{Sn}_{x} \mathrm{Se}^{118} x=0.31, g_{v}=4, m_{t}^{-}=0.143 m_{0}, m_{l}^{-}=2.0 m_{0}$,

$m_{t}^{+}=0.167 m_{0}, m_{l}^{+}=0.286 m_{0}, P_{\|}=3.2 \times 10^{-10} \mathrm{eV} \mathrm{m}, P_{\perp}=4.1 \times 10^{-10} \mathrm{eV} \mathrm{m}$,

$g_{v}=4$, and $E_{g}=0.137 \mathrm{eV}$

(1) Stressed $n$-InSb: ${ }^{95} m^{*}=0.048 m_{0}, E_{g}=0.081 \mathrm{eV}, B_{2}^{*}=14 \times 10^{-11} \mathrm{eV} \mathrm{m}$,

$C_{1}^{c}=4 \mathrm{eV}, C_{2}^{c}=3 \mathrm{eV}, a_{0}=-10 \mathrm{eV}, b_{0}=-2 \mathrm{eV}, d=-5 \mathrm{eV}, S_{11}=0.6$

$\times 10^{-3}(\mathrm{kbar})^{-1}, S_{22}=0.42 \times 10^{-3}(\mathrm{kbar})^{-1}, S_{33}=0.39 \times 10^{-3}(\mathrm{kbar})^{-1}$, and $S_{12}=0.5 \times 10^{-3}(\mathrm{kbar})^{-1}$

(m) $n$-GaP: ${ }^{96} m_{\|}^{*}=0.92 m_{0}, \wp=1, k_{0}=1.7 \times 10^{19} \mathrm{~m}^{-1}, V_{G}=0.21 \mathrm{eV}, m_{\perp}^{*}$

$=0.25 m_{0}$, and $g_{v}=6$

(n) $\mathrm{PtSb}_{2}:{ }^{110} \lambda_{1}=0.02 \mathrm{eV}, l_{1}=0.32 \mathrm{eV}, \nu_{1}=0.39 \mathrm{eV}, n_{1}=0.65 \mathrm{eV}, a$

$=6.43 \AA, I_{0}=0.30(\mathrm{eV})^{2}, \delta_{0}^{\prime}=0.02 \mathrm{eV}$, and $g_{v}=6$

(o) $n$-GaSb: ${ }^{111} E_{g}=0.81 \mathrm{eV}, \Delta=0.80 \mathrm{eV}, P=9.48 \times 10^{-10} \mathrm{eV} \mathrm{m}, \overline{\mathrm{s}}_{0}=-2.1$, $\bar{v}_{0}=-1.49$, and $\bar{\omega}_{0}=0.42$

(p) Bismuth: ${ }^{107} E_{g}=0.0153 \mathrm{eV}, m_{1}=0.00581 m_{0}, m_{2}=1.25 m_{0}, m_{3}$

$=0.0113 m_{0}, m_{2}^{\prime}=0.36 m_{0}, M_{2}=0.128 m_{0}, M_{2}^{\prime}=0.80 m_{0}, g_{v}=3$, and $g_{s}=2$

tions. In addition, the results of this article find two specific applications, namely the suggestions for the experimental determinations of the Einstein relation for the diffusivity-tomobility ratio, and the carrier contribution to the elastic constants for 1D systems having arbitrary carrier energy spectra in the realm of nanoscience.

\section{RESULTS AND DISCUSSION}

Using Eqs. (5), (13), and (14) and Table I for numerical values of the energy band constants, in Figs. 1(a) and 1(b) we have plotted the TPM for QWs of $n-\mathrm{CdGeAs}_{2}$ and $n-\mathrm{Cd}_{3} \mathrm{As}_{2}$ as a function of film thickness, at low temperatures, where the quantum effects become prominent, in which curves $(a)$ and $(b)$ have been drawn corresponding to $\delta=0$ and $\delta \neq 0$, respectively, for the purpose of assessing the influence of crystal-field splitting on the TPM in QWs of both materials. The TPM in accordance with the three-band model of Kane (curve $c$, for the purpose of assessing the combined influence of the crystal-field splitting and the anisotropies of the effective electron mass and the spin-orbit splitting of the valance bands), the two-band model of Kane (curve $d$, to assess the influence of the spin-orbit splitting constant), and the parabolic energy bands (curve $e$, to assess the influence of band nonparabolicity) have been drawn for relative assessment. In Figs. 1(c) and 1(d), we have drawn the TPM as a function of $n_{1 \mathrm{D}}$ for all the cases of Figs. 1(a) and 1(b). Using Eq. (5) together with Eqs. (3), (6), (4), and 


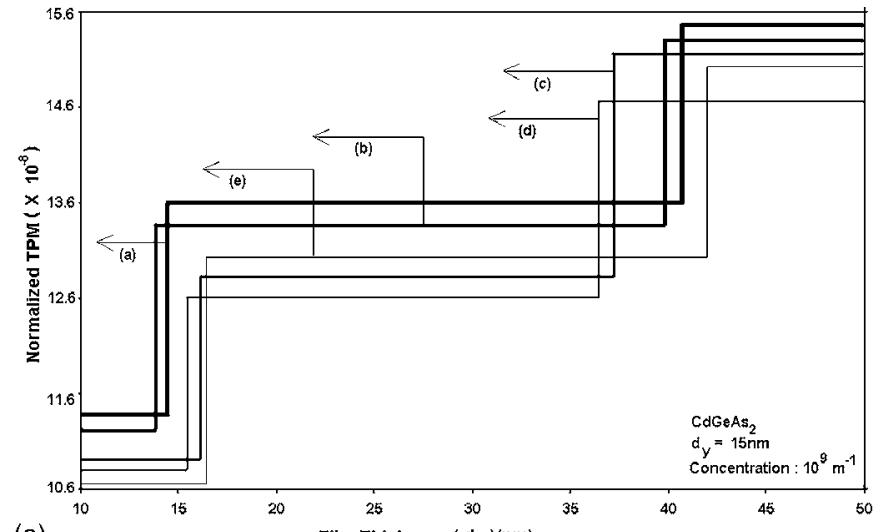

(a)
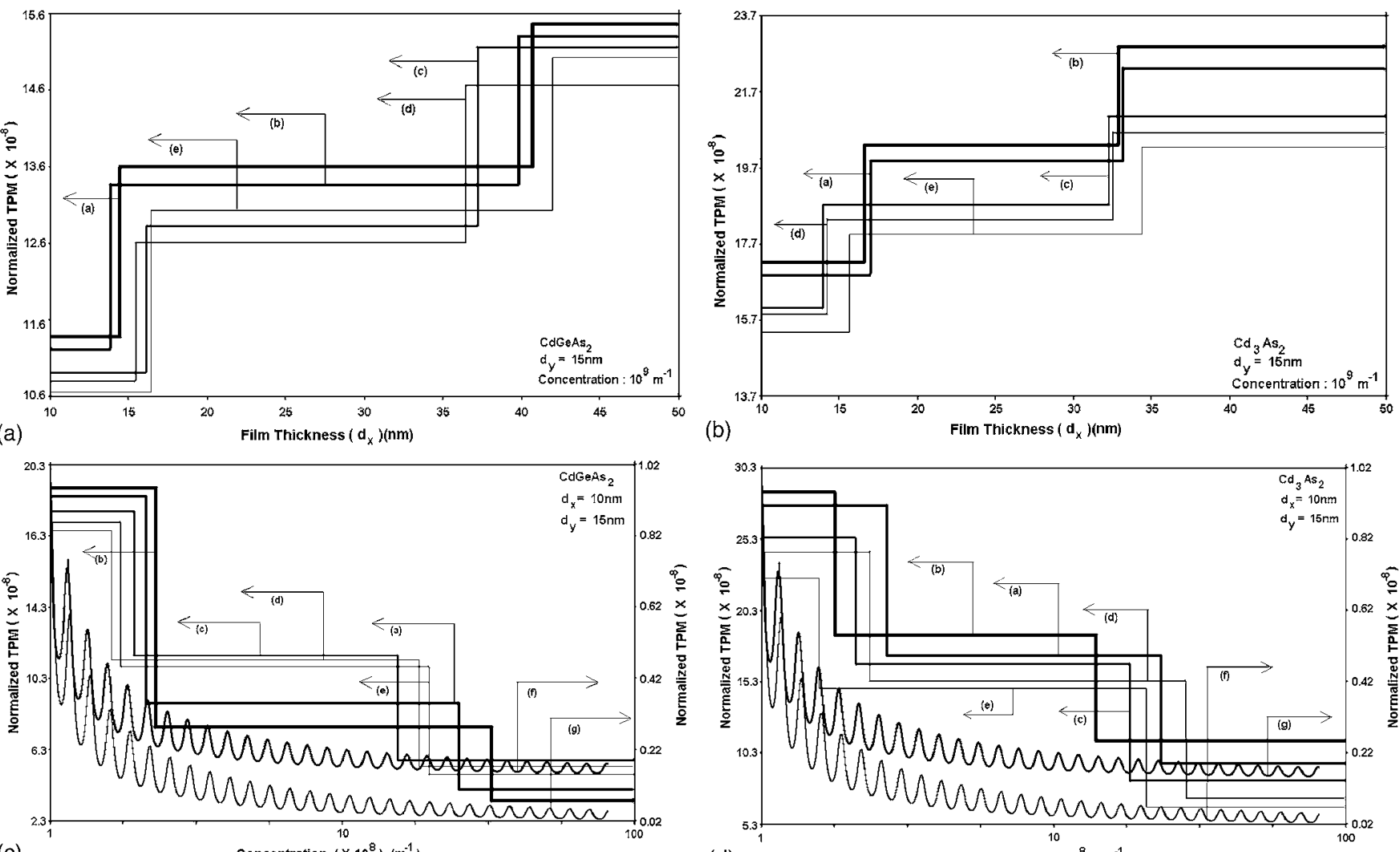

(b)

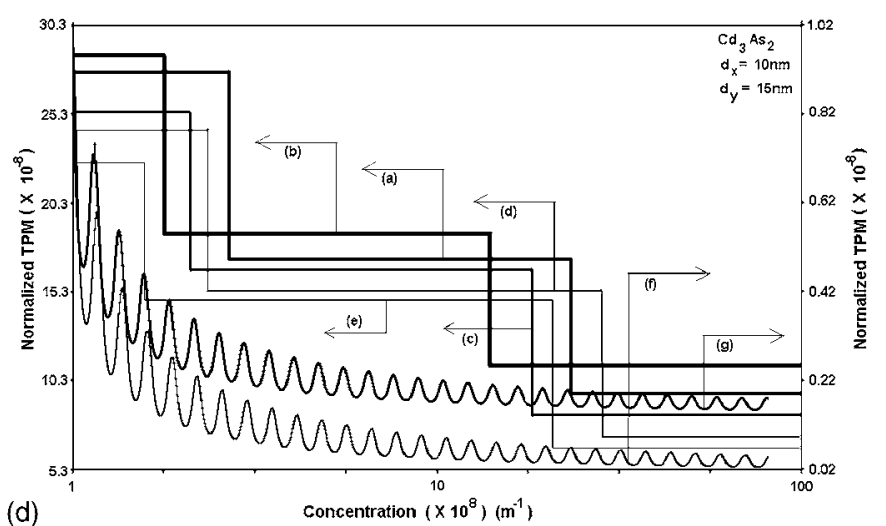

(c)

Concentration $\left(\times 10^{8}\right)\left(\mathrm{m}^{-1}\right)$

(d)

Concentration $\left(\times 10^{8}\right)\left(\mathrm{m}^{-1}\right)$

FIG. 1. (a) Plot of the TPM as a function of film thickness for the QWs of $n-\mathrm{CdGeAs}_{2}$ in accordance with $(a)$ the generalized proposed band model with $\delta=0$, (b) the generalized band model with $\delta \neq 0,(c)$ the simplified three-band model of Kane, where, $m^{*}=\left[\left(m_{\|}^{*}+m_{\perp}^{*}\right) / 2\right]$ and $\Delta_{=}=\left[\left(\Delta_{\|}^{*}+\Delta_{\perp}^{*}\right) / 2\right],(d)$ the two-band model of Kane, and (e) the parabolic energy bands. (b) Plot of the TPM as a function of film thickness for the QWs of $n-\mathrm{Cd}_{3} \mathrm{As}_{2}$ in accordance with $(a)$ the generalized proposed band model with $\delta=0,(b)$ the generalized band model with $\delta \neq 0,(c)$ the simplified three-band model of Kane, where $m^{*}=\left[\left(m_{\|}^{*}\right.\right.$ $\left.\left.+m_{\perp}^{*}\right) / 2\right]$ and $\Delta=\left[\left(\Delta_{\|}^{*}+\Delta_{\perp}^{*}\right) / 2\right],(d)$ the two-band model of Kane, and (e) the parabolic energy bands. (c) Plot of the TPM as a function of electron concentration per unit length for the QWs of $n$-CdGeAs 2 in accordance with $(a)$ the generalized proposed band model with $\delta=0$, (b) the generalized band model with $\delta \neq 0,(c)$ the three-band model of Kane, where $m^{*}=\left[\left(m_{\|}^{*}+m_{\perp}^{*}\right) / 2\right]$ and $\Delta=\left[\left(\Delta_{\|}^{*}+\Delta_{\perp}^{*}\right) / 2\right],(d)$ the two-band model of Kane, and $(e)$ the parabolic energy bands. The curves $(f)$ and $(g)$ exhibit the corresponding plots for $(n, n)$ and $(n, 0)$ CNTs, respectively. (d) Plot of the TPM as a function of electron concentration per unit length for the QWs of $n-\mathrm{Cd}_{3} \mathrm{As}_{2}$ in accordance with $(a)$ the generalized proposed band model with $\delta=0,(b)$ the generalized band model with $\delta \neq 0,(c)$ the three-band model of Kane, where $m^{*}=\left[\left(m_{\|}^{*}+m_{\perp}^{*}\right) / 2\right]$ and $\Delta=\left[\left(\Delta_{\|}^{*}+\Delta_{\perp}^{*}\right) / 2\right],(d)$ the two-band model of Kane, and $(e)$ the parabolic energy bands. The curves $(f)$ and $(g)$ exhibit the corresponding plots for $(n, n)$ and $(n, 0)$ CNTs, respectively.

(7), we have superimposed the plots of the TPM for the ( $n$, $n)$ and $(n, 0)$ carbon nanotubes as a function of $n_{1 \mathrm{D}}$ as shown by curves $(f)$ and $(g)$ of Figs. 1(c) and 1(d), respectively, for the purpose of relative comparison.

It appears that the TPM increases with the increasing film thickness in quantum steps with increasing step length for the said materials. Furthermore, for both the $(n, n)$ and ( $n, 0)$ CNTs, the TPM is a decreasing oscillatory function with increasing electron statistics, and the magnitude of the TPM is lower than that of the TPM in QWs, which is apparent from both Figs. 1(c) and 1(d) which show the characteristic features of CNTs. The influence of quantum confinement is immediately apparent from the all the curves of Figs. 1(a)-1(d), since the TPM depends strongly on the film thickness and electron concentration per unit length in a step-like manner, which is in direct contrast with the corresponding bulk specimens. Moreover, the TPM can become several orders of magnitude lower than that of the corresponding bulk specimens of the same materials, which is also a direct signature of quantum confinement. It appears from the curves (a)-(e) of Figs. 1(c) and 1(d) that the TPM decreases with the increasing $n_{1 \mathrm{D}}$ in a step-like manner for all types of band models as considered here, although the widely different numerical values are determined by the constants of the energy spectra. The step functional dependence is due to the crossing over of the Fermi level by the quantized subbands. For each coincidence of a quantized level with the Fermi level, there would be a discontinuity in the density-of-states function resulting in a change of the height of the steps. With large values of film thickness, the height of the steps decreases and the TPM decreases with increasing electron statistics in a nonoscillatory manner. The height of the step and the rate of increment are totally determined by the band structure. The influence of crystal-field splitting is immediately apparent from comparing curves $(a)$ and $(b)$ for QWs of both $n$-CdGeAs 2 and $n-\mathrm{Cd}_{3} \mathrm{As}_{2}$ from Figs. 1(a)-1(d). The crystal-field splitting decreases the numerical values of the TPM in both cases. The numerical values of the TPM in accordance with the three-band model of Kane is greater than that of the corresponding two-band model, which reflects the fact that the presence of the spin-orbit splitting constants enhance the magnitude of the TPM in both cases. It may be noted that the presence of the band nonparabolicity in accordance with the two-band model of Kane enhances 

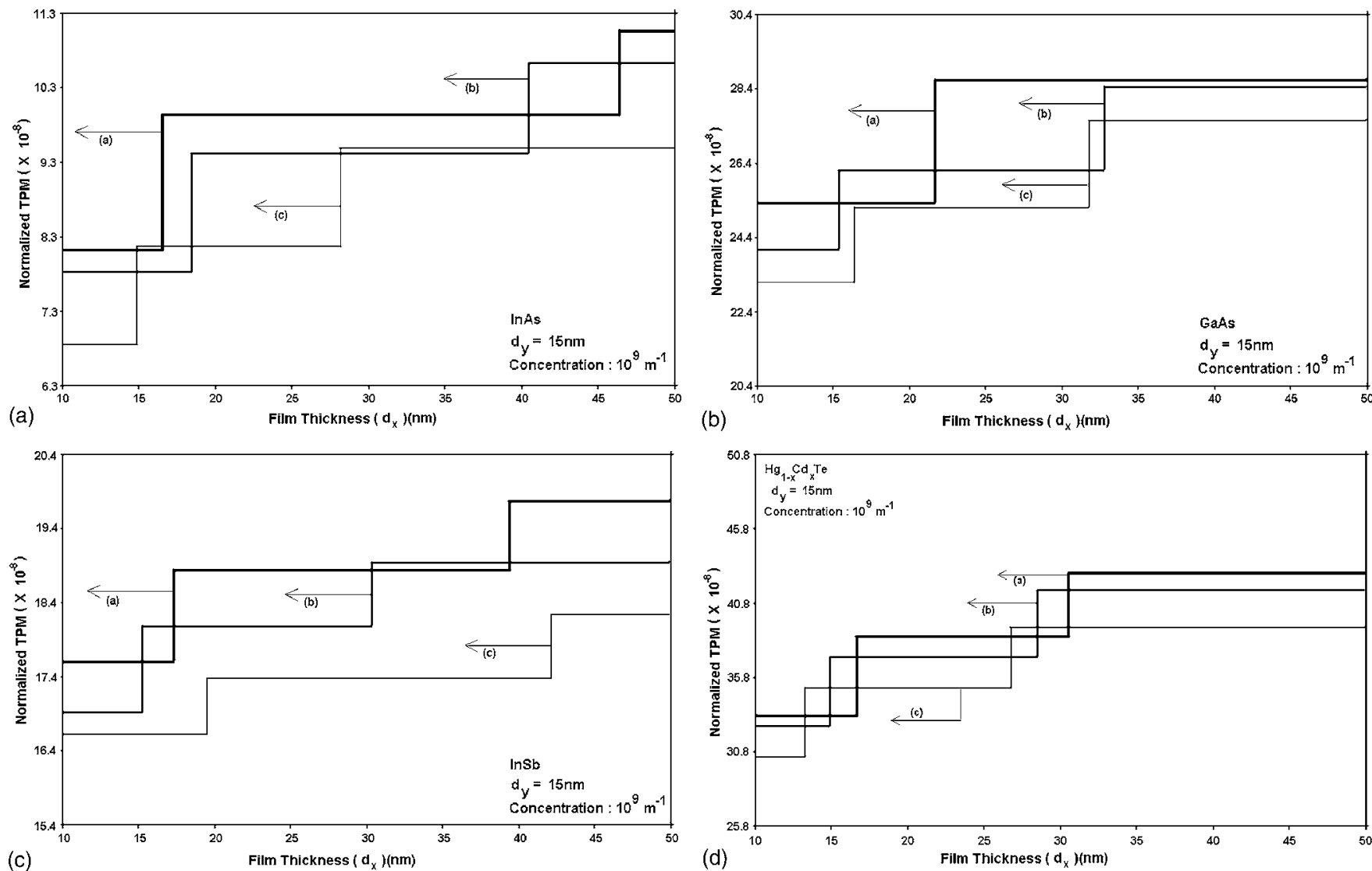

(c)

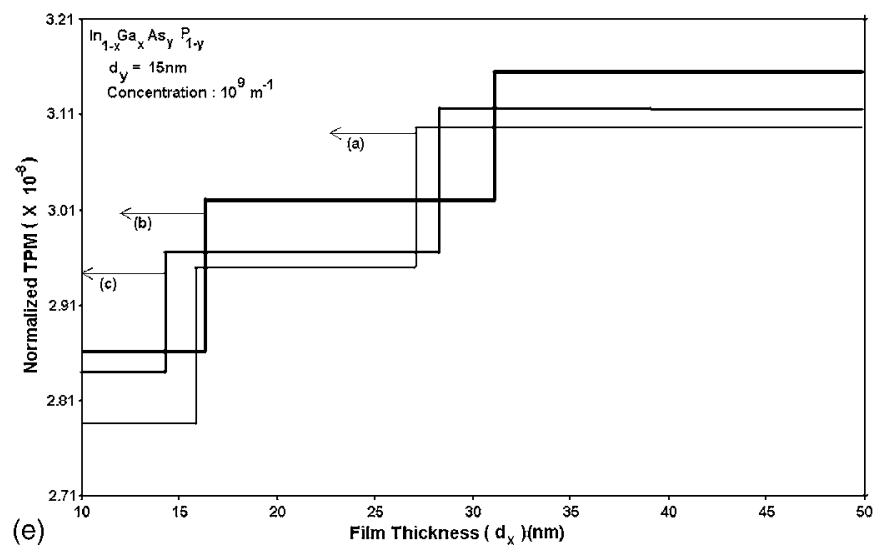

FIG. 2. (a) Plot of the TPM as a function of film thickness for the QWs of $n$-InAs in accordance with $(a)$ the three-band model of Kane, $(b)$ the two-band model of Kane, and $(c)$ the parabolic energy bands. (b) Plot of the TPM as a function of film thickness for the QWs of $n$-GaAs in accordance with $(a)$ the three-band model of Kane, $(b)$ the two-band model of Kane, and $(c)$ the parabolic energy bands. (c) Plot of the TPM as a function of film thickness for the QWs of $n$-InSb in accordance with (a) the three-band model of Kane, $(b)$ the two-band model of Kane, and $(c)$ the parabolic energy bands. (d) Plot of the TPM as a function of film thickness for the QWs of $\mathrm{Hg}_{1-x} \mathrm{Cd}_{x} \mathrm{Te}$ in accordance with $(a)$ the three-band model of Kane, $(b)$ the two-band model of Kane, and $(c)$ the parabolic energy bands. (e) Plot of the TPM as a function of film thickness for the QWs of $\operatorname{In}_{1-x} \mathrm{Ga}_{x} \mathrm{As}_{y} \mathrm{P}_{1-y}$ lattice matched to InP in accordance with $(a)$ the three-band model of Kane, $(b)$ the two-band model of Kane, and $(c)$ the parabolic energy bands.

the peaks of the steps for TPM for all cases of quantum confinement. The appearance of the humps of the respective curves is due to the redistribution of the electrons among the quantized energy levels when the quantum numbers corresponding to the highest occupied level change from one fixed value to the other. With varying electron concentration, a change is reflected in the TPM through the redistribution of the electrons among the quantized levels. Although the TPM varies with all the variables in all the limiting cases, as is evident from all the curves of Figs. 1(a)-1(d), the rates of variations are totally band-structure-dependent. Thus, for two types of one-dimensional systems, the natures of the variation of the TPM are also different, which exhibits the signatures of the two entirely different two-dimensional quantum confinements.

Using Eqs. (17), (18), (20), (21), (23), and (24), in Figs. 2(a)-2(e) we have plotted the TPM as a function of the film thickness for QWs of $n$-InAs, $n$-GaAs, $n$-InSb, $\mathrm{Hg}_{1-x} \mathrm{Cd}_{x} \mathrm{Te}$, and $\mathrm{In}_{1-x} \mathrm{Ga}_{x} \mathrm{As}_{y} \mathrm{P}_{1-y}$ lattice matched to $\mathrm{InP}$, in which the plots $(a),(b)$, and $(c)$ represent the three-band model of Kane, the two-band model of Kane, and that of the parabolic energy band, respectively. Figures 2(a)-2(e) have further 

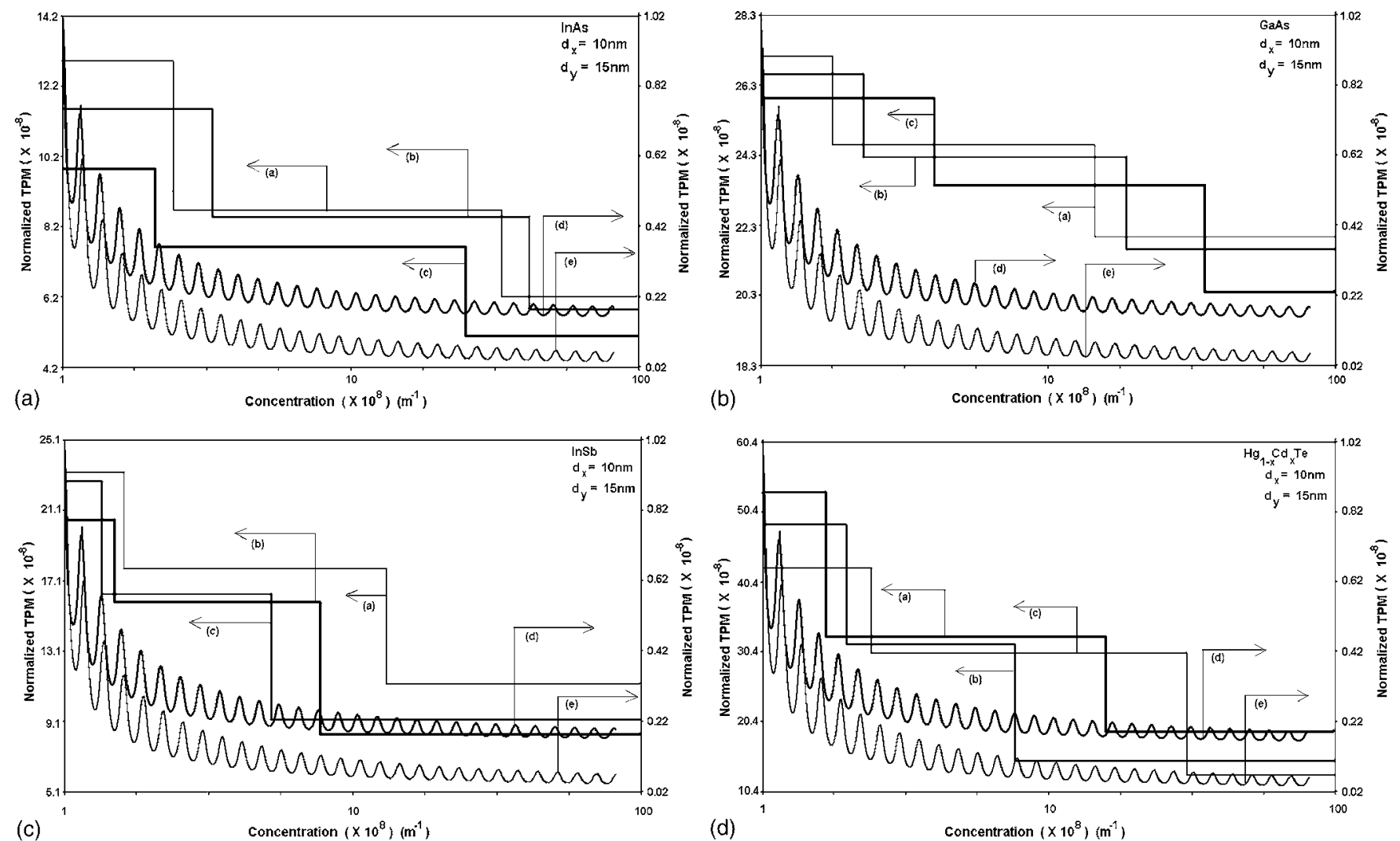

(c)

Concentration $\left(\times 10^{8}\right)\left(\mathrm{m}^{-1}\right)$

(d)

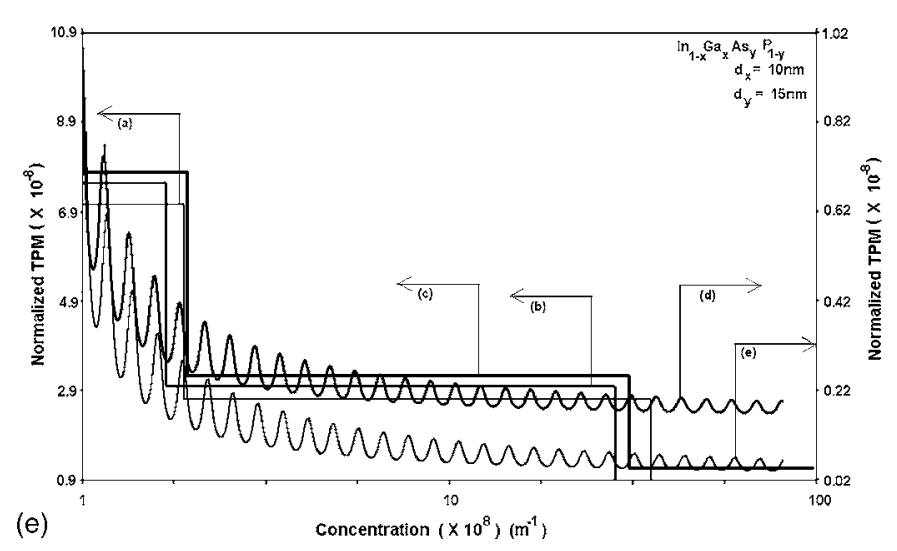

FIG. 3. (a) Plot of the TPM as a function of electron concentration per unit length for the QWs of $n$-InAs in accordance with (a) the three-band model of Kane, (b) the two-band model of Kane, and $(c)$ the parabolic energy bands. The curves $(d)$ and $(e)$ are the corresponding plots for $(n, n)$ and $(n, 0)$ CNTs. (b) Plot of the TPM as a function of electron concentration per unit length for the QWs of $n$-GaAs in accordance with $(a)$ the three-band model of Kane, $(b)$ the two-band model of Kane, and $(c)$ the parabolic energy bands. The curves $(d)$ and $(e)$ exhibit the corresponding plots for $(n$, $n)$ and $(n, 0)$ CNTs, respectively. (c) Plot of the TPM as a function of electron concentration per unit length for the QWs of $n$-InSb in accordance with $(a)$ the three-band model of Kane, $(b)$ the two-band model of Kane, and $(c)$ the parabolic energy bands. The curves $(d)$ and $(e)$ exhibit the corresponding plots for ( $n, n)$ and $(n, 0)$ CNTs, respectively. (d) Plot of the TPM as a function of electron concentration per unit length for the $\mathrm{QWs}_{\mathrm{s}} \mathrm{Hg}_{1-x} \mathrm{Cd}_{x} \mathrm{Te}_{\mathrm{in}}$ accordance with $(a)$ the three-band model of Kane, $(b)$ the two-band model of Kane, and $(c)$ the parabolic energy bands. The curves $(d)$ and $(e)$ exhibit the corresponding plots for $(n, n)$ and $(n, 0)$ CNTs, respectively. (e) Plot of the TPM as a function of electron concentration per unit length for the $\mathrm{QWs}$ of $\mathrm{In}_{1-x} \mathrm{Ga}_{x} \mathrm{As}_{y} \mathrm{P}_{1-y}$ lattice matched to InP in accordance with $(a)$ the three-band model of Kane, $(b)$ the two-band model of Kane, and $(c)$ the parabolic energy bands. The curves $(d)$ and (e) exhibit the corresponding plots for $(n, n)$ and $(n, 0) \mathrm{CNTs}$, respectively.

been drawn as a function of electron concentration per unit length for all the above-mentioned materials in Figs. 3(a)-3(e), respectively, where we have superimposed the plots of the TPM for the CNTs for the purpose of relative comparisons. In Figs. 1(a)-3(e), it appears that the numerical values of the TPM in QWs are greatest for the ternary compounds. The quantum jump in QWs is asymmetric as compared with the corresponding symmetric and systematic periodic oscillations of the TPM for CNTs.

Using Eqs. (30), (31), (34), and (35), in Figs. 4(a) and 4(b) we have plotted the TPM as a function of film thickness and electron concentration per unit length for QWs of II-VI and IV-VI materials, respectively, where the plot $(b)$ refers to $p$-CdS in accordance with the Hopfield model with $C_{0}$ $\neq 0 \mathrm{eV} \mathrm{m}$, while the plot $(a)$ corresponds to the same for $C_{0}=0 \mathrm{eV} \mathrm{m}$ for the purpose of assessing the influence of the splitting of the two-spin states by the spin-orbit coupling and the crystalline field in this case. It appears from the above figures that the influence of the term $C_{0}$ is the increment of 

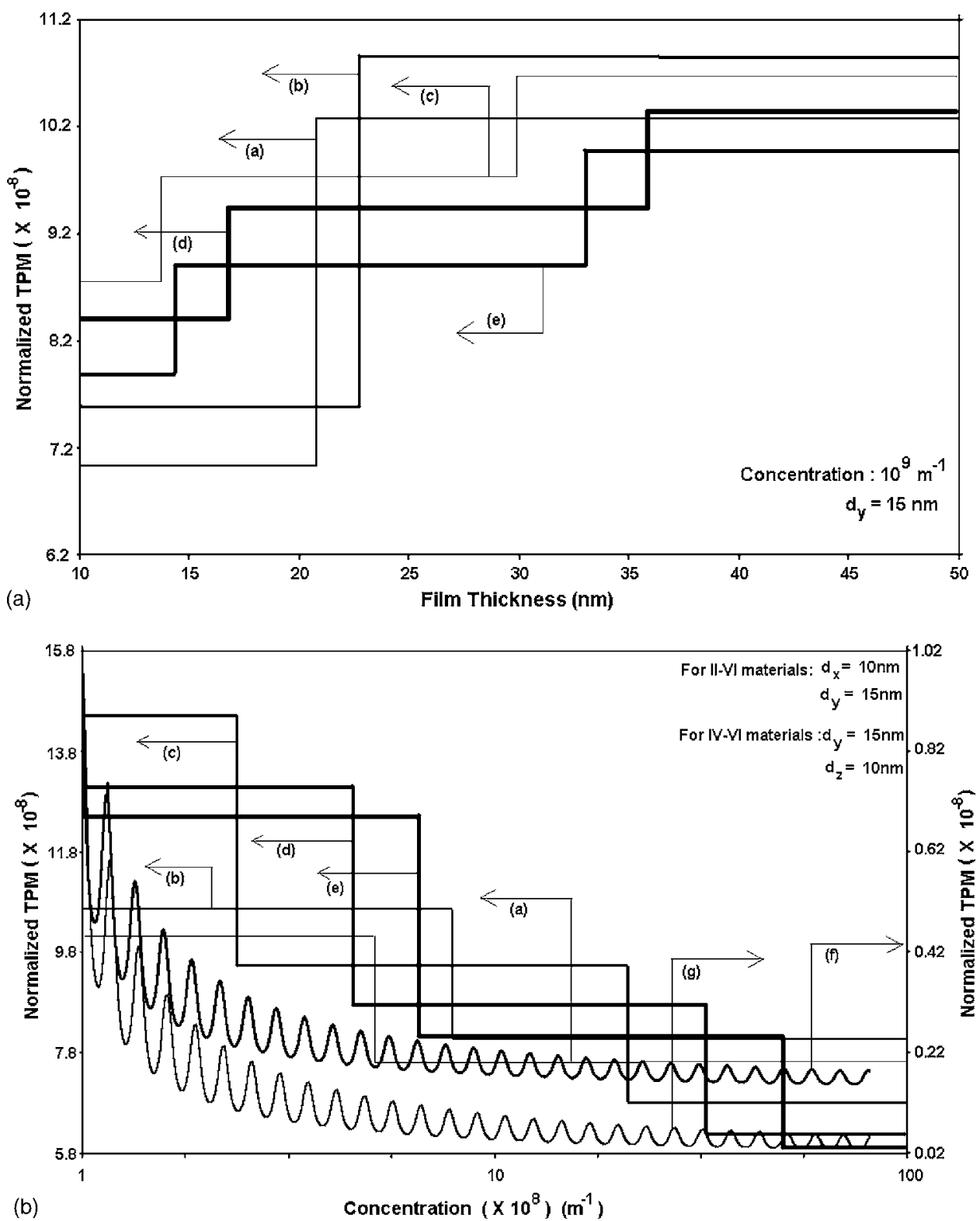

FIG. 4. (a) Plot of the TPM as a function of film thickness for the QWs of $\mathrm{CdS}$ in accordance with the Hopfield model where $(a) C_{0}=0$ and $(b) C_{0}$ $\neq 0$. The curves $(c),(d)$, and $(e)$ exhibit the TPM for the QWs of $n$-PbTe, $\mathrm{PbSnTe}$, and $\mathrm{Pb}_{1-x} \mathrm{Sn}_{x} \mathrm{Se}$, respectively. (b) Plot of the TPM as a function of electron concentration per unit length for the QWs of CdS in accordance with the Hopfield model where $(a)$ $C_{0}=0$ and $(b) C_{0} \neq 0$. The curves $(c)$, $(d)$, and (e) exhibit the TPM for QWs of $n$-PbTe, PbSnTe, and $\mathrm{Pb}_{1-x} \mathrm{Sn}_{x} \mathrm{Se}$. The curves $(f)$ and $(g)$ exhibit the corresponding plots for $(n, n)$ and $(n, 0)$ CNTs, respectively. the quantum jumps of the oscillatory 1D TPM for QWs of II-VI materials. From the superposition of the corresponding TPM for CNTs, one can infer the relative difference in magnitude and the nature of oscillations in this case. The curves $(c), \quad(d)$, and (e) refer to $n$-PbTe, $n$-PbSnTe, and $n$ - $\mathrm{Pb}_{1-x} \mathrm{Sn}_{x} \mathrm{Se}$, respectively. From Fig. 4(b), the influence of the energy band parameters on the TPM for the three different QWs of IV-VI materials as considered here and the relative comparison with that in CNTs are apparent from all the plots.

Using Eqs. (38), (39), (42), (43), (47), (48), (52), and (53), we have plotted in Figs. 5(a) and 5(b) the TPM as a function of film thickness and electron concentration per unit length for QWs of stressed $n-\operatorname{InSb}[(a)$ and $(b)$ represent the plots both in the presence and absence of stress], $n$-GaP [curve $(c)$ ], $\mathrm{PtSb}_{2}$ [curve $(d)$ ], and $n$-GaSb [curve $(e)$ ], respectively. For stressed $n$-InSb, in the presence of the stress, the magnitude of the TPM is being decreased as compared with the same under stress-free conditions. It is important to note that the numerical values of the TPM in CNTs are less than that in QWs of stressed materials.
The signatures of the totally two different types of TPM in CNTs and QWs are apparent by comparing all the curves of Figs. 5(a) and 5(b), respectively. Figures 6(a) and 6(b) exhibit the TPM as a function of film thickness and electron concentration per unit length for all models of QWs of bismuth. The relative difference in magnitude and the nature of oscillations can be assessed from all the figures and the superposition of the corresponding TPMs for CNTs in this case.

Since the experimental curves of $n_{1 \mathrm{D}}$ versus $G$ are not available in the literature to the best of our knowledge for the present generalized systems, we cannot compare our theoretical formulation with the experimental results, although the generalized analysis as presented in this context can be checked when the experimental results of $G$ for the present generalized systems would appear. If the direction normal to the film were taken differently from that as assumed in this work, the expressions for the TPM in quasi-one-dimensional structures would be different analytically, since the basic dispersion laws of many important materials are anisotropic. In formulating the generalized electron energy spectrum for 

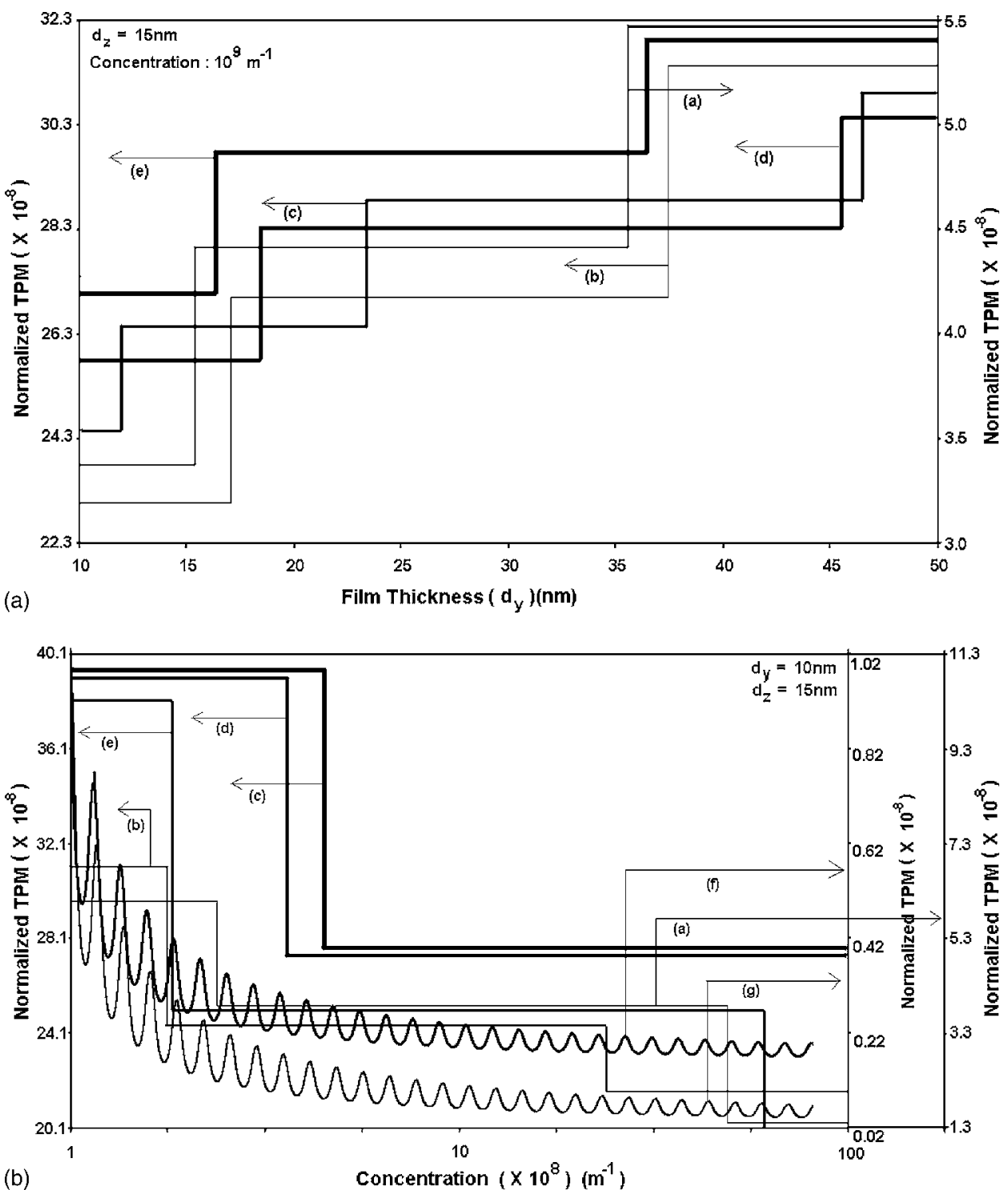

FIG. 5. (a) Plot of the TPM as a function of film thickness for the QWs of stressed InSb in accordance with $(a)$ the absence of stress and $(b)$ the presence of stress. The curves $(c),(d)$, and (e) exhibit the TPM for QWs of $n$-GaP, $n-\mathrm{PtSb}_{2}$, and $n$-GaSb, respectively. (b) Plot of the TPM as a function of electron concentration per unit length for the QWs of stressed InSb in accordance with $(a)$ the absence of stress and $(b)$ the presence of stress. The curves $(c),(d)$, and $(e)$ exhibit the TPM in QWs of $n$-GaP, $n$ - $\mathrm{PtSb}_{2}$, and $n$-GaSb, respectively. The curves $(f)$ and $(g)$ are the corresponding plots for $(n, n)$ and $(n, 0)$ CNTs, respectively. nonlinear optical materials, we have considered the crystalfield splitting, the anisotropies in the momentum-matrix elements, and the spin-orbit splitting constants, respectively. In the absence of the crystal-field splitting constant together with the assumptions of isotropic effective electron mass and isotropic spin-orbit splitting, our basic relation as given by Eq. (12) converts into Eq. (15). Equation (15) is the wellknown three-band model of Kane ${ }^{109}$ and is valid for III-V compounds, in general. It should be used as such for studying the electronic properties of $n$-InAs where the spin-orbit splitting parameter $(\Delta)$ is of the order of the band gap $\left(E_{g}\right)$. For many important materials, $\Delta \gg E_{g}$ or $\Delta \ll E_{g}$, and under these inequalities Eq. (15) assumes the form $E\left(1+E E_{g}^{-1}\right)$ $=\hbar^{2} k^{2} / 2 m^{*}$, which is the well-known two-band Kane model. ${ }^{109}$ Also under the condition $E_{g} \rightarrow \infty$, the above equation gets simplified to the well-known form of parabolic energy bands as $E=\hbar^{2} k^{2} / 2 m^{*}$. The TPM has been studied for QWs of II-VI, IV-VI, stressed materials, $n$-GaP, $p$-PtSb $\mathrm{Pb}_{2}$, and $n$-GaSb compounds in accordance with the models of Hopfield, ${ }^{93}$ Dimmock, ${ }^{94}$ Seiler, ${ }^{95}$ Rees, ${ }^{96}$ Emtage, ${ }^{110}$ and Seiler, ${ }^{111}$ respectively. Because of the importance of bismuth in the whole field of nanoscience, we have further studied the
TPM in bismuth in accordance with the McClure and Choi model, ${ }^{107}$ the Hybrid model, ${ }^{106}$ the Cohen model, ${ }^{105}$ the Lax model, ${ }^{99}$ and the ellipsoidal parabolic model, ${ }^{98}$ respectively.

It is important to note that under certain limiting conditions, all the results for all the models derived here have transformed into the well-known classical TPM equation valid for nondegenerate semiconductors having parabolic energy bands. This indirect test not only exhibits the mathematical compatibility of our formulation, but it also reflects the fact that our simple analysis is a more generalized one, since one can obtain the corresponding results for relatively wide gap materials having parabolic energy bands under certain limiting conditions from our present generalized analysis.

We have not considered other types of compounds or external physical variables for numerical computations in order to keep the presentation brief. With different sets of energy band parameters, we shall get different numerical values of the TPM, though the nature of variations of the TPM as shown here would be similar for other types of materials, and the simplified analysis of this article exhibits the basic qualitative features of the TPM for such compounds. In this con- 

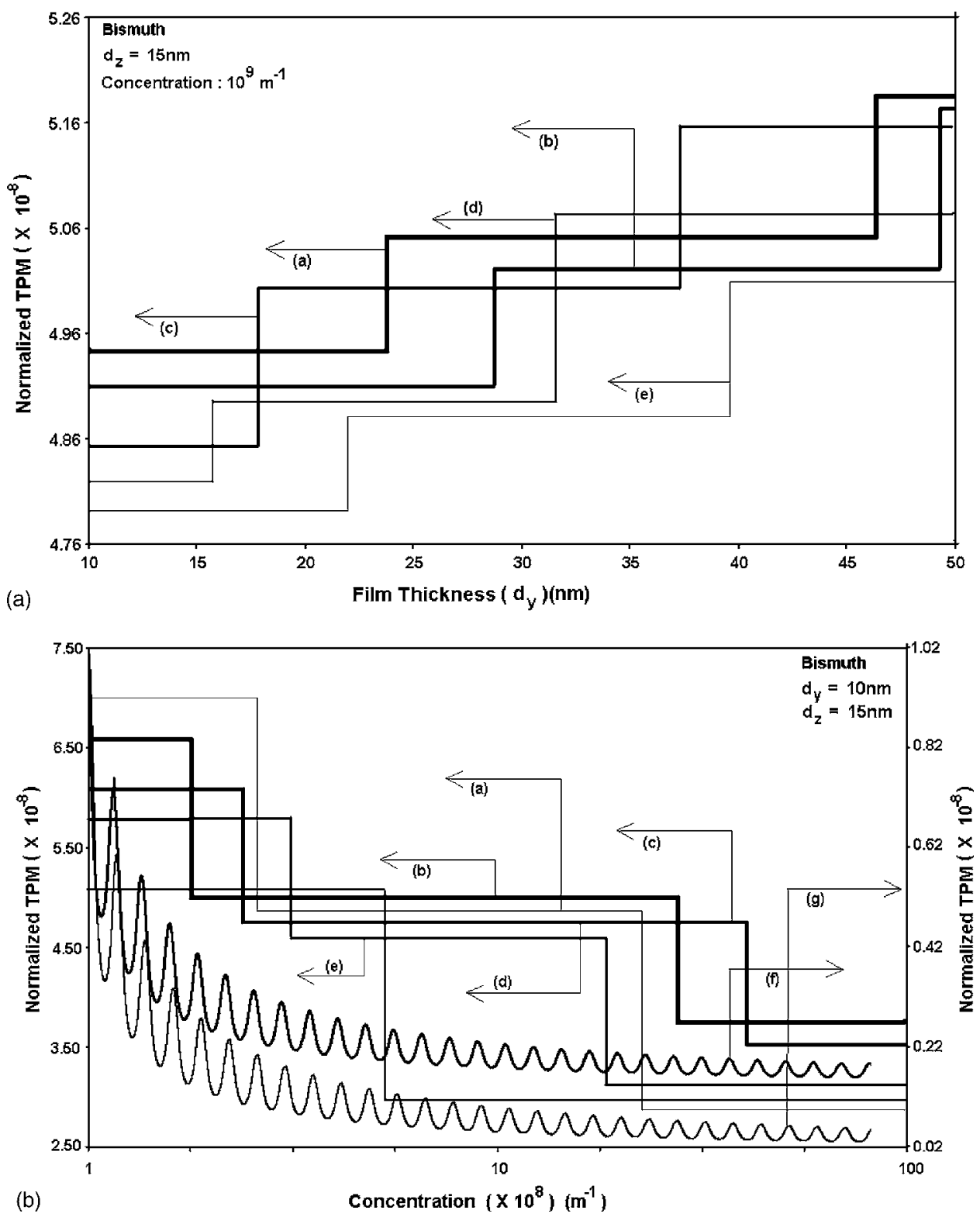

FIG. 6. (a) Plots of the TPM as a function of film thickness for QWs of $\mathrm{Bi}$ in accordance with $(a)$ the McClure and Choi model, (b) the Hybrid model, (c) the Cohen model, $(d)$ the Lax model, and (e) the parabolic ellipsoidal model. (b) Plots of the TPM as a function of $n_{1 \mathrm{D}}$ for QWs of $\mathrm{Bi}$ in accordance with $(a)$ the McClure and Choi model, (b) the Hybrid model, (c) the Cohen model, $(d)$ the Lax model, and (e) the parabolic ellipsoidal. The curves $(f)$ and $(g)$ exhibit the TPM for the $(n, n)$ and $(n, 0)$ CNTs, respectively. text, it may be pointed out that we have not considered the many body and the hot-electron effects in this simplified theoretical formalism due to the absence of proper analytical techniques for including them for the present generalized system as considered in this article. Our simplified approach will be useful for the purpose of relative comparison when the methods for tackling the formidable problem of inclusion of the many-body and hot-electron effects for the present generalized system would appear. The inclusion of the above-mentioned effects would certainly increase the accuracy of the results, although our suggestions for the experimental determinations of the Einstein relation for the diffusivity-to-mobility ratio and the carrier contribution to the elastic constants are independent of incorporating the above-mentioned effects, and the qualitative features of the 1D TPM would not change in the presence of the aforementioned effects.

It must be mentioned that a direct research application of the quantized materials is in the area of band structure, and the study of transport phenomena and the formulation of the electronic properties of nanocompounds are based on the dispersion relations in such materials. The theoretical results of our paper can be used to determine the TPM of the constituent bulk materials in the absence of quantum effects. The objective of the present work is not solely to investigate the 1D TPM in various cases, but also to suggest experimental determinations of the Einstein relation and the carrier contribution to the elastic constants for materials having arbitrary band structures, which, in turn, are again dimensionindependent. Finally, it may be noted that the purpose of this article is not solely to study the signatures of the two entirely different 1D quantized materials on the TPM for CNTs and QWs of nonlinear optical materials, ternary, quaternary, IIVI, IV-VI, stressed materials, GaP, $p$-PtSb $2, n-\mathrm{GaSb}$, and $\mathrm{Bi}$, but also to formulate the appropriate electron statistics in its most generalized form, since the transport and the related phenomena in 1D systems having different band structures are based on the temperature-dependent electron statistics in such materials. 
${ }^{1}$ S. Iijima, Nature (London) 354, 56 (1991).

${ }^{2}$ V. N. Popov, Mater. Sci. Eng., R. 43, 61 (2004).

${ }^{3}$ J. Sandler, M. S. P. Shaffer, T. Prasse, W. Bauhofer, K. Schulte, and A. H. Windle, Polymer 40, 5967 (1999).

${ }^{4}$ D. Qian, E. C. Dickey, R. Andrews, and T. Rantell, Appl. Phys. Lett. 76, 2868 (2000).

${ }^{5}$ J. Kong, N. R. Franklin, C. W. Zhou, M. G. Chapline, S. Peng, K. J. Cho, and H. J. Dai, Science 287, 622 (2000).

${ }^{6}$ W. A. Deheer, A. Chatelain, and D. Ugarte, Science 270, 1179 (1995).

${ }^{7}$ A. G. Rinzler, J. H. Hafner, P. Nikolaev, L. Lou, S. G. Kim, D. Tomanek, P. Nordlander, D. T. Olbert, and R. E. Smalley, Science 269, 1550 (1995).

${ }^{8}$ A. C. Dillon, K. M. Jones, T. A. Bekkedahl, C. H. Kiang, D. S. Bethune, and M. J. Heben, Nature (London) 386, 377 (1997).

${ }^{9}$ C. Liu, Y. Y. Fan, M. Liu, H. T. Cong, H. M. Cheng, and M. S. Dresselhaus, Science 286, 1127 (1999).

${ }^{10}$ P. Kim and C. M. Lieber, Science 286, 2148 (1999).

${ }^{11}$ D. Srivastava, Nanotechnology 8, 186 (1997).

${ }^{12}$ C. Ke and H. D. Espinosa, Appl. Phys. Lett. 85, 681 (2004).

${ }^{13}$ J. W. Kang and H. J. Hwang, Nanotechnology 15, 1633 (2004); J. Cumings and A. Zettl, Science 289, 602 (2000).

${ }^{14}$ S. J. Tans, M. H. Devoret, H. J. Dai, A. Thess, R. E. Smalley, L. J. Geerligs, and C. Dekker, Nature (London) 386, 474 (1997).

${ }^{15}$ S. J. Tans, A. R. M. Verschueren, and C. Dekker, Nature (London) 393, 49 (1998).

${ }^{16}$ P. Avouris, Acc. Chem. Res. 35, 1026 (2002).

${ }^{17}$ P. G. Collins, A. Zettl, H. Bando, A. Thess, and R. E. Smalley, Science 278, 100 (1997).

${ }^{18}$ R. H. Baughman, A. A. Zakhidov, and W. A. de Heer, Science 297, 787 (2002).

${ }^{19}$ Z. Yao, H. W. C. Postma, L. Balents, and C. Dekker, Nature (London) 402, 273 (1999).

${ }^{20}$ S. J. Tans, A. R. M. Verschueren, and C. Dekker, Nature (London) 393, 49 (1998).

${ }^{21}$ T. Rueckes, K. Kim, E. Joselevich, G. Y. Tseng, C. L. Cheung, and C. M. Lieber, Science 289, 94 (2000).

${ }^{22}$ A. Bachtold, P. Hadley, T. Nakanish, and C. Dekker, Science 294, 1317 (2001).

${ }^{23}$ H. Sasaki, Jpn. J. Appl. Phys. 19, 94 (1980).

${ }^{24}$ P. M. Petroff, A. C. Gossard, R. A. Logan, and W. Weigmann, Appl. Phys Lett. 41, 635 (1982)

${ }^{25}$ I. Miller, A. Miller, A. Shahar, U. Koren, and P. J. Corvini, Appl. Phys Lett. 54, 188 (1989).

${ }^{26}$ F. Sols, M. Macucci, U. Ravaioli, and K. Hess, Appl. Phys. Lett. 54, 350 (1989).

${ }^{27}$ C. S. Lent and D. J. Kirkner, J. Appl. Phys. 67, 6353 (1990).

${ }^{28}$ C. S. Kim, A. M. Satanin, Y. S. Joe, and R. M. Cosby, Phys. Rev. B 60, 10962 (1999).

${ }^{29}$ S. Midgley and J. B. Wang, Phys. Rev. B 64, 153304 (2001).

${ }^{30}$ T. Sugaya, J. P. Bird, M. Ogura, Y. Sugiyama, D. K. Ferry, and K. Y. Jang, Appl. Phys. Lett. 80, 434 (2002).

${ }^{31}$ B. Kane, G. Facer, A. Dzurak, N. Lumpkin, R. Clark, L. PfeiKer, and K. West, Appl. Phys. Lett. 72, 3506 (1998).

${ }^{32}$ C. Dekker, Phys. Today 52, 22 (1999).

${ }^{33}$ A. Yacoby, H. L. Stormer, N. S. Wingreen, L. N. Pfeiffer, K. W. Baldwin, and K. W. West, Phys. Rev. Lett. 77, 4612 (1996).

${ }^{34}$ Y. Hayamizu, M. Yoshita, S. Watanabe, H. A. L. Pfeiker, and K. West, Appl. Phys. Lett. 81, 4937 (2002).

${ }^{35}$ S. Frank, P. Poncharal, Z. L. Wang, and W. A. Heer, Science 280, 1744 (1998).

${ }^{36}$ I. Kamiya, I. I. Tanaka, K. Tanaka, F. Yamada, Y. Shinozuka, and H. Sakaki, Physica E (Amsterdam) 13, 131 (2002).

${ }^{37}$ A. K. Geim, P. C. Main, N. LaScala, L. Eaves, T. J. Foster, P. H. Beton, J. W. Sakai, F. W. Sheard, M. Henini, and G. Hill, Phys. Rev. Lett. 72, 2061 (1994).

${ }^{38}$ A. S. Mel'nikov and V. M. Vinokur, Nature (London) 415, 60 (2002).

${ }^{39}$ K. Schwab, E. A. Henriksen, J. M. Worlock, and M. L. Roukes, Nature (London) 404, 974 (2000).

${ }^{40}$ L. Kouwenhoven, Nature (London) 403, 374 (2000).

${ }^{41}$ S. Komiyama, O. Astafiev, V. Antonov, and H. Hirai, Nature (London) 403, 405 (2000).

${ }^{42}$ E. Paspalakis, Z. Kis, E. Voutsinas, and A. F. Terziz, Phys. Rev. B 69, 155316 (2004)

${ }^{43}$ J. H. Jefferson, M. Fearn, D. L. J. Tipton, and T. P. Spiller, Phys. Rev. A 66, 042328 (2002).
${ }^{44}$ J. Appenzeller, C. Schroer, T. Schapers, A. Hart, A. Froster, B. Lengeler, and H. Luth, Phys. Rev. B 53, 9959 (1996).

${ }^{45}$ J. Appenzeller and C. Schroer, J. Appl. Phys. 87, 3165 (2000).

${ }^{46}$ P. Debray, O. E. Raichev, M. Rahman, R. Akis, and W. C. Mitchel, Appl. Phys. Lett. 74, 768 (1999).

${ }^{47}$ P. M. Solomon, Proc. IEEE 70, 489 (1982); T. E. Schlesinger and T. Kuech, Appl. Phys. Lett. 49, 519 (1986).

${ }^{48}$ H. Heiblum, D. C. Thomas, C. M. Knoedler, and M. I. Nathan, Appl. Phys. Lett. 47, 1105 (1985).

${ }^{49}$ O. Aina, M. Mattingly, F. Y. Juan, and P. K. Bhattacharyya, Appl. Phys. Lett. 50, 43 (1987).

${ }^{50}$ I. Suemune and L. A. Coldren, IEEE J. Quantum Electron. 24, 1778 (1988).

${ }^{51}$ D. A. B. Miller, D. S. Chemla, T. C. Damen, J. H. Wood, A. C. Burrus, A. C. Gossard, and W. Weigmann, IEEE J. Quantum Electron. 21, 1462 (1985).

${ }^{52}$ J. Hajdu and R. Landwher, Springer Ser. Solid-State Sci. 57, 97 (1985).

${ }^{53}$ K. von Klitzing, Rev. Mod. Phys. 58, 519 (1986).

${ }^{54}$ K. P. Ghatak and S. N. Biswas, J. Appl. Phys. 70, 299 (1991).

${ }^{55}$ B. Mitra and K. P. Ghatak, Phys. Lett. A 141, 81 (1989).

${ }^{56}$ W. Zawadzki, Adv. Phys. 23, 435 (1974).

${ }^{57}$ F. M. Peeters and P. Vasilopoulos, Phys. Rev. B 46, 4667 (1992).

${ }^{58}$ K. P. Ghatak, M. Mitra, B. Goswami, and B. Nag, Mol. Cryst. Liq. Cryst. Sci. Technol., Sect. B: Nonlinear Opt. 16, 167 (1996).

${ }^{59}$ I. M. Tsidilkovskii, Band Structures of Semiconductors (Pergamon, London, 1982).

${ }^{60}$ S. K. Biswas, A. R. Ghatak, A. Neogi, A. Sharma, S. Bhattacharya, and K. P. Ghatak, Physica E (Amsterdam) 36, 163 (2007).

${ }^{61}$ S. P. Zelenim, A. S. Kondratev, and A. E. Kuchma, Sov. Phy. Semicond. 16, 355 (1982).

${ }^{62}$ K. P. Ghatak and B. Nag, Nanostruct. Mater. 2, 14 (1995).

${ }^{63}$ B. M. Askerov, N. F. Gashimzade, and M. M. Panakhov, Sov. Phys. Solid State 29, 456 (1987)

${ }^{64}$ J. L. Shay and J. W. Wernik, Ternary Chalcopyrite Compounds: Growth, Electronic Properties and Applications (Pergamon, London, 1975).

${ }^{65}$ G. P. Chuiko, Sov. Phys. Semicond. 15, 739 (1981).

${ }^{66}$ E. K. Arushanov, A. F. Knyazev, A. N. Nateprov, and S. I. Radautsan, Sov. Phys. Semicond. 15, 828 (1981).

${ }^{67}$ S. I. Radautsan, E. K. Arushanov, A. N. Nateprov, and G. P. Chuiko, Cadmium Arsenide and Phosphide (Shtiintsa, Kishinev, 1976) (in Russian).

${ }^{68}$ I. W. Rowe and J. L. Shay, Phys. Rev. 83, 451 (1971).

${ }^{69}$ I. J. Hopfdeld, Phys. Chem. Solids 15, 97 (1960).

${ }^{70}$ A. Shileika, Surf. Sci. 37, 730 (1973); J. L. Shay and B. Tell, ibid. 37, 748 (1973).

${ }^{71}$ B. Kh. Mamedov and E. O. Osmanov, Sov. Phys. Semicond. 5, 1120 (1972).

${ }^{72}$ O. V. Emelyanenko, F. P. Kesamanly, I. K. Polushina, and V. A. Skripkin, Sov. Phys. Semicond. 5, 304 (1971).

${ }^{73}$ S. D. Chemla, F. R. Beglay, and L. R. Byer, IEEE J. Quantum Electron. 10, 71 (1974).

${ }^{74}$ H. Kildal, Phys. Rev. 10, B5082 (1974).

${ }^{75}$ G. P. Chuiko and N. N. Chuiko, Sov. Phys. Semicond. 15, 739 (1981); K. P. Ghatak and S. N. Biswas, Proc. SPIE 1484, 146 (1991).

${ }^{76}$ R. Bisaro, G. Laurencin, A. Friederich, and M. Razeghi, Appl. Phys. Lett. 40, 978 (1982).

${ }^{77}$ R. C. Alferness, C. H. Joyner, M. D. Divino, M. J. R. Martyak, and L. L. Buhl, Appl. Phys. Lett. 49, 125 (1986).

${ }^{78}$ T. E. Bell, IEEE Spectrum 20, 38 (1983).

${ }^{79}$ P. Y. Lu, C. H. Wang, C. M. Williams, S. N. G. Chu, and C. M. Stiles, Appl. Phys. Lett. 49, 1372 (1986)

${ }^{80}$ N. R. Taskar, I. B. Bhat, K. K. Parat, D. H. Terry Ehsani, and S. K. Gandhi, J. Vac. Sci. Technol. A 7, 281 (1989).

${ }^{81}$ E. Weiss and N. Mainzer, J. Vac. Sci. Technol. A 7, 391 (1989).

${ }^{82}$ F. Koch, Springer Series in Solid State Sciences (Springer-Verlag, Berlin, 1984), Vol. 53.

${ }^{83}$ D. H. Jaw, D. S. Caoand, and G. B. Stringfellow, J. Appl. Phys. 69, 2552 (1991).

${ }^{84}$ M. L. Timmons, S. Bedair, M. R. J. Markunas, and J. A. Hutchby, Proceedings of the 16th IEEE Photovoltaic Specialists Conferences (IEEE, San Diego, 1982), p. 666.

${ }^{85}$ M. K. Lee, D. S. Wu, and H. H. Tung, J. Appl. Phys. 62, 3209 (1987).

${ }^{86}$ K. P. Ghatak and S. Bhattacharya, J. Appl. Phys. 102, 073704 (2007).

${ }^{87}$ S. Adachi, J. Appl. Phys. 53, 8775 (1982). 
${ }^{88}$ S. I. Radaustan, V. I. Morozava, A. F. Knyazev, L. S. Koval, E. K. Arushanov, and A. N. Nataprov, Sov. Phys. Semicond. 19, 691 (1985).

${ }^{89}$ M. A. Herman and M. Pessa, J. Appl. Phys. 57, 2671 (1985).

${ }^{90}$ K. Sakamoto and Y. Okabe, J. Appl. Phys. 23, 444 (1986).

${ }^{91}$ T. Nguyen Duy, J. Meslage, and G. Pichard, J. Cryst. Growth 72, 490 (1985).

${ }^{92}$ D. H. Chow and T. C. Mc Gill, Appl. Phys. Lett. 48, 1485 (1986).

${ }^{93}$ J. J. Hopfield, J. Appl. Phys. 32, 2277 (1961).

${ }^{94}$ J. O. Dimmock, in The Physics of Semimetals and Narrow Gap Compounds, edited by D L. Carter and R. T. Bates (Pergamon, Oxford, 1971), p. 319.

${ }^{95}$ D. G. Seiler, B. D. Bajaj, and A. E. Stephens, Phys. Rev. B 16, 2822 (1977); K. P. Ghatak and B. Mitra, Phys. Scr. 46, 182 (1992).

${ }^{96} \mathrm{G}$. J. Rees, Physics of Compounds, in Proceedings of the 13th International Conference, edited by F. G. Fumi (North Holland, Amsterdam, 1976), p. 1166

${ }^{97}$ D. Shoenberg, Proc. R. Soc. London, Ser. A 170, 341 (1939).

${ }^{98}$ B. Abeles and S. Mieboom, Phys. Rev. 101, 544 (1956).

${ }^{99}$ B. Lax, J. G. Mavroides, H. J. Ziegeer, and R. Keyes, Phys. Rev. Lett. 5, 241 (1960).

${ }^{100}$ M. Maltz and M. S. Dresselhaus, Phys. Rev. B 2, 2877 (1970).

${ }^{101}$ M. Cankurtaran, M. Celik, and T. Alper, J. Phys. F: Met. Phys. 16, 853 (1986).

${ }^{102}$ Y. H. Kao, Phys. Rev. 129, 1122 (1963).

${ }^{103}$ R. J. Dinger and A. W. Lawson, Phys. Rev. B 3, 253 (1971).
${ }^{104}$ J. A. Koch and J. D. Jensen, Phys. Rev. 184, 643 (1969).

${ }^{105}$ M. H. Cohen, Phys. Rev. 121, 387 (1961); J. Rose and R. Schuchardt, Phys. Status Solidi B 117, 213 (1983).

${ }^{106}$ S. Takaoka, H. Kawamura, K. Murse, and S. Takano, Phys. Rev. B 13, 1428 (1976).

${ }^{107}$ J. W. McClure and K. H. Choi, Solid State Commun. 21, 1015 (1977).

${ }^{108}$ X. Yang and J. Ni, Phys. Rev. B 72, 195426 (2005); D. Kahn and J. P. Lu, ibid. 60, 6535 (1999).

${ }^{109}$ B. R. Nag, Electron Transport in Compound Semiconductors (SpringerVerlag, Berlin, 1980).

${ }^{110}$ P. R. Emtage, Phys. Rev. 138, A246 (1965).

${ }^{111}$ D. G. Seiler, W. M. Beeker, and L. M. Roth, Phys. Rev. B 1, 764 (1970).

${ }^{112}$ W. Szuszkiewicz, Phys. Status Solidi B 79, 691 (1977).

${ }^{113}$ P.T. Landsberg, Eur. J. Phys. 2, 213 (1981), and references cited therein.

${ }^{114}$ S. N. Mohammad, J. Phys. C 13, 2685 (1980).

${ }^{115}$ H. Kroemer, IEEE Trans. Electron Devices 25, 850 (1978); S. Mukherjee, S. N. Mitra, P. K. Bose, A. R. Ghatak, A. Neogi, J. P. Banerjee, A. Sinha, M. Pal, S. Bhattacharya, and K. P. Ghatak, J. Comput. Theor. Nanosci. 4, 550 (2007).

${ }^{116}$ R. W. Keyes, IBM. J. Res. Dev. 5, 266 (1961)

${ }^{117}$ K. P. Ghatak, S. Bhattacharya, S. K. Biswas, A. Dey, and A. K. Dasgupta, Phys. Scr. 75, 820 (2007).

${ }^{118}$ O. Madelung, Semiconductors: Data Handbook, 3rd ed. (Springer, Berlin, 2004); M. Kriechbaum, P. Kocevar, H. Pascher, and G. Bauer, IEEE J. Quantum Electron. 24, 1727 (1988). 